\title{
Momentum-Space Spin Texture in a Topological Superconductor
}

\author{
Florian Loder ${ }^{1,2}$, Arno P. Kampf ${ }^{2}$, Thilo Kopp ${ }^{1}$, and Daniel Braak ${ }^{1}$ \\ Center for Electronic Correlations and Magnetism, \\ ${ }^{1}$ Experimental Physics VI, ${ }^{2}$ Theoretical Physics III \\ Institute of Physics, University of Augsburg, 86135 Augsburg, Germany
}

(Dated: January 3, 2017)

\begin{abstract}
A conventional superconductor with spin-orbit coupling turns into a topological superconductor beyond a critical strength of the Zeeman energy. The spin-expectation values $\boldsymbol{S}(\boldsymbol{k})$ in momentum space trace this transition via a characteristic change in the topological character of the spin texture within the Brillouin zone. At the transition the skyrmion counting number switches from 0 to $1 / 2$ identifying the topological superconductor via its meron-like spin texture. The change in the skyrmion counting number is crucially controlled by singular points of the map $\boldsymbol{S}(\boldsymbol{k}) /|\boldsymbol{S}(\boldsymbol{k})|$ from the Brillouin zone, i.e. a torus, to the unit sphere. The complexity of this spin-map is discussed at zero temperature as well as for the extension to finite temperatures.
\end{abstract}

\section{INTRODUCTION}

The classification of electronic states by integer numbers that are not quantum numbers but derive from the topology of the entire electronic system has become a significant concept in the past two decades [1-4]. Whereas the quantum numbers may depend on the details of the realization of a complex system, the topological character is considered to be robust. The topology is a global property of the entire set of the system's quantum states. In many electronic systems, the robustness results from an energy gap that separates the occupied from the unoccupied states. Closing and reopening this gap by the variation of control parameters may allow for a transition into a topologically different state. Solids that are insulators or superconductors can be classified accordingly. A prominent example for a topological insulator is the quantum Hall state, where the number of occupied Landau levels defines its topological character [5]. Thouless, Kohmoto, Nightingale, and den Nijs showed that the corresponding topological number is obtained as an integral of the Berry curvature over the Brillouin zone (BZ) [6]. It is typically referred to as the TKNN number $C_{\text {TKNN }}$. In mathematically precise terms the curvature is the first Chern class and $C_{\text {TKNN }}$ the first Chern number of the principal $U(1)$ bundle of wave functions over the torus $T^{2}$ of momentum eigenstates in the BZ [7].

Also a nodeless superconductor can be characterized by a topological invariant. Topologically similar to the quantum Hall state is a two-dimensional (2D) $s$-wave superconductor with a Zeeman coupling to a sufficiently strong magnetic field in the presence of a Rashba spinorbit coupling (SOC) [8-13]. The same physics applies to topological superfluidity in polarized ultracold atomic Fermi gases with SOC [14-19]. The topological $s$-wave superconductor is sometimes referred to as an effective $p$-wave superconductor, because its pairing amplitude in the two spin-orbit split bands acquires the antisymmetric wavevector-dependence of the Rashba SOC [9]. Nevertheless, its topological properties are different from those of a true triplet $p$-wave superconductor. In particular, whereas the $s$-wave superconductor remains topologically trivial in the absence of a magnetic field, the $p$-wave superconductor itself has a non-trivial topology, which is not captured by $C_{\mathrm{TKNN}}$, but rather by a $\mathbb{Z}_{2}$ topological quantum number $C_{\mathbb{Z}_{2}}$ that applies to time-reversal invariant systems [20-27].

While the non-trivial topology of the $p$-wave state is intrinsic to the spin structure defined by the superconducting (SC) order parameter (OP), a topologically nontrivial character of a conventional $s$-wave superconductor requires that the normal conducting state already exhibits a specific spin structure in momentum space. This spin texture is imposed by the SOC; its relation to the topological character is, however, by no means obvious. To analyze the emergence of the topological SC state the Chern and the skyrmion numbers was previously introduced also in Ref. [28].

However, although the spin texture in momentum space is reminiscent of a skyrmion, its pattern might not completely cover the full solid angle of all possible spin orientations. If the texture covers half of the full solid angle, it may be interpreted as a kind of "half skyrmion", which for spatial spin patterns has been called a meron [29]. But is the spin structure of such a meron characterized by a well-defined topological invariant? The resolution of this fundamental issue is provided by our analysis. We find that the mapping of the BZ (torus) to the hemisphere of the normalized spin expectation values becomes singular at a finite number of points in the BZ; the analysis of these singular points allows us to prove the topological character of the meron spin structure.

These findings naturally pose the question, if and how the topological character of the $s$-wave superconductor is also reflected in its finite temperature behavior. The Berry curvature and the respective Chern number are, by construction, meant to reveal intrinsic features of the system's groundstate and therefore they are unsuited to address finite temperatures. Moreover, a topological invariant, such as the skyrmion number, cannot reflect a smooth evolution with temperature as the topological in- 
variant is constrained to integer numbers. We investigate instead if the Hall conductance or deliberately selected measures of the spin texture provide tools to continuously follow the evolution from the topological ground state to the canonical ensemble at finite temperatures. Specifically, we identify a peculiar spin product which serves this purpose. At zero temperature it is equivalent to the Berry curvature but, since it is based on expectation values of spin operators, this spin product is straightforwardly extended to finite temperatures.

For a superconductor with SOC the orientation of the external magnetic field matters, and we therefore also examine the momentum-space spin texture upon field rotation. As a sufficiently strong in-plane component of the field induces finite-momentum pairing, this analysis is intricate. Yet, we show that the crucial vortex structures, which are attached to the singular points in the spin map from the BZ to the unit sphere, are preserved also for an in-plane magnetic field component. The meron character of the spin texture in the 2D topological superconductor is therefore maintained, when the magnetic field is rotated from an out-of-plane to an in-plane orientation.

\section{TOPOLOGICAL CONCEPTS}

Before analyzing the invariants for a topological superconductor we first recollect some of the basic concepts for topological insulators, which the subsequent sections build on. Two-band insulators are straightforward generalizations of two-level systems which can be written in terms of pseudospins represented by Pauli matrices in the Hamiltonian. The real-valued Bloch vector $\boldsymbol{h}$ controls the rotation in the pseudospin space.

The two-band insulator is a paradigmatic electronic system to introduce topological quantum numbers. In this section we relate seemingly different topological invariants of the electronic state. Specifically we will address how the Chern number is related to the Brouwer degree of a map from a torus $T^{2}$ to a unit sphere $S^{2}$ and, moreover, how this is related to the spin texture in momentum space and the associated skyrmion number. Eventually, the Chern number may be identified as the TKNN number which can be derived from a Kubo formula for the Hall conductance. These different topological aspects have been discussed in several textbooks (see, for example, the books by Thouless [1], Bernevig [2], Volovik [29], Jost [30], and references therein). In this section we shall connect the aspects raised above. It is Stokes' theorem for multiply connected surfaces which is pivotal in deriving these relations, especially the connection between the Chern number and the Brouwer degree of the map generated by the normalized Bloch vector [31].

In two space dimensions we consider the two-band lattice Hamiltonian, diagonal in momentum space,

$$
H=\sum_{\boldsymbol{k}}\left[\epsilon_{\boldsymbol{k}} \sigma^{0}+\boldsymbol{h}(\boldsymbol{k}) \cdot \boldsymbol{\sigma}\right]_{s s^{\prime}} c_{\boldsymbol{k} s}^{\dagger} c_{\boldsymbol{k} s^{\prime}}
$$

The pseudospin indices $s, s^{\prime}$ for the electron creation and annihilation operators refer to a discrete degree of freedom. Later on we will specify their actual nature (spin and/or orbital degrees of freedom); $\boldsymbol{\sigma}$ denotes the vector of Pauli matrices. The Bloch vector $\boldsymbol{h}(\boldsymbol{k})$ is assumed to be a smooth, periodic, nowhere vanishing function of the wavevector $\boldsymbol{k}=\left(k_{1}, k_{2}\right)^{\top}$, where $\boldsymbol{k}$ varies through the two-dimensional Brillouin zone $-\pi \leq k_{j} \leq \pi$.

It describes therefore a smooth map from the torus $T^{2}$ (a compact manifold without boundary) into $\mathbb{R}^{3}$. The normalized Bloch vector $\boldsymbol{h}(\boldsymbol{k}) / h(\boldsymbol{k})$ with $h(\boldsymbol{k})=|\boldsymbol{h}(\boldsymbol{k})|$ is a map from $T^{2}$ into the unit sphere $S^{2}$. The eigenenergies of the two bands are $\xi_{ \pm, \boldsymbol{k}}=\epsilon_{\boldsymbol{k}} \pm|\boldsymbol{h}(\boldsymbol{k})|$ and the normalized single-particle eigenstates of $H$ are

$$
\boldsymbol{u}_{ \pm}(\boldsymbol{k})=\frac{1}{\sqrt{2 h\left(h \pm h_{z}\right)}}\left(h_{z} \pm h, h_{x}+i h_{y}\right)^{\top} .
$$

Here, \pm denotes the band index for the diagonalized Hamiltonian. The $\boldsymbol{k}$ dependence of $h$ and $h_{x}, h_{y}, h_{z}$ on the right hand side has been suppressed. In the following we consider the insulating case of a completely filled lower band $\boldsymbol{u}_{-}(\boldsymbol{k})=\left(u_{1}(\boldsymbol{k}), u_{2}(\boldsymbol{k})\right)^{\top}$ and an empty upper band $\boldsymbol{u}_{+}(\boldsymbol{k})$.

\section{A. Berry connection and Berry curvature}

The Berry connection of the lower band is defined as the 1-form

$$
\boldsymbol{v}=i \boldsymbol{u}_{-}^{\dagger}\left(\partial_{k_{1}} \boldsymbol{u}_{-}\right) \mathrm{d} k_{1}+i \boldsymbol{u}_{-}^{\dagger}\left(\partial_{k_{2}} \boldsymbol{u}_{-}\right) \mathrm{d} k_{2} .
$$

The 1-form $\boldsymbol{v}$ is real-valued due to the normalization of $\boldsymbol{u}_{-}$and $\boldsymbol{u}_{-}^{\dagger}=\left(u_{1}^{*}, u_{2}^{*}\right)^{\top}$. The Berry phase picked up along a path $\gamma$ in the $\mathrm{BZ}$ is

$$
\phi(\gamma)=\int_{\gamma} \boldsymbol{v} .
$$

Specifically for the lower-band eigenstates in Eq. (2), with $\boldsymbol{v}=v_{1} \mathrm{~d} k_{1}+v_{2} \mathrm{~d} k_{2}$ and $\partial_{k_{j}}=\partial_{j}$, we obtain

$$
v_{j}=R^{-2}\left(h_{y} \partial_{j} h_{x}-h_{x} \partial_{j} h_{y}\right), \quad R=\sqrt{2 h\left(h-h_{z}\right)} .
$$

$\boldsymbol{v}$ is a scalar-valued 1-form, that is, each component $v_{j}$ transforms as a scalar $U(1)$ gauge field because the wavefunctions $\boldsymbol{u}_{-}(\boldsymbol{k})$ are sections of a complex line bundle over the torus. The associated curvature is therefore the exterior derivative [30],

$$
\boldsymbol{\Omega}=\mathrm{d} \boldsymbol{v}=\left(\partial_{1} v_{2}-\partial_{2} v_{1}\right) \mathrm{d} k_{1} \wedge \mathrm{d} k_{2} .
$$

With $\left\langle\boldsymbol{u}_{-}\left|\nabla_{\boldsymbol{k}}\right| \boldsymbol{u}_{-}\right\rangle=\left(\left\langle\boldsymbol{u}_{-}\left|\partial_{1}\right| \boldsymbol{u}_{-}\right\rangle,\left\langle\boldsymbol{u}_{-}\left|\partial_{2}\right| \boldsymbol{u}_{-}\right\rangle, 0\right)^{\top}$, we may write the value $\Omega(\boldsymbol{k})$ of $\boldsymbol{\Omega}$ at $\boldsymbol{k}$ in the familiar vectorial notation [33],

$$
\Omega(\boldsymbol{k})=i\left[\nabla_{\boldsymbol{k}} \times\left\langle\boldsymbol{u}_{-}\left|\nabla_{\boldsymbol{k}}\right| \boldsymbol{u}_{-}\right\rangle\right]_{z} .
$$

The 2 -form $\boldsymbol{\Omega} /(2 \pi)$ is the first (and top) Chern class, an element of the second cohomology group $H^{2}\left(T^{2}\right)$ of 
the torus [32]. Its integral over the $\mathrm{BZ}$ yields the first Chern number of the $U(1)$-bundle represented by $\boldsymbol{v}$. If $\boldsymbol{v}$ were a smooth function throughout the $B Z$, this integral would necessarily vanish. But as seen from Eq. (5), $\boldsymbol{v}$ has isolated singular points, if $\boldsymbol{h}=h_{z} \boldsymbol{e}_{z}$ with $h_{z}>0$, that is, whenever the point $\boldsymbol{k}$ is mapped by $\boldsymbol{h} / h$ to the north pole of $S^{2}$ where $R(\boldsymbol{k})=0$. In this case one has to apply Stokes' theorem for multiply connected surfaces [31],

$$
\int_{B Z} \boldsymbol{\Omega}=\oint_{\partial B Z} \boldsymbol{v}-\sum_{l} \oint_{c_{l}} \boldsymbol{v}=-\sum_{l} \oint_{c_{l}} \boldsymbol{v}
$$

The paths $c_{l}$ denote infinitesimally small circles around the singular points $\boldsymbol{k}_{l}$ with $R\left(\boldsymbol{k}_{l}\right)=0$ which constitute punctures in the base manifold (that is, the BZ-torus).

We show in Appendix A that Eq. (8) measures a topological property of the map $\boldsymbol{h}(\boldsymbol{k})$,

$$
\int_{B Z} \boldsymbol{\Omega}=2 \pi \sum_{l} \operatorname{sign}\left(J\left(\boldsymbol{k}_{l}\right)\right)=2 \pi C_{\text {Brouwer }} .
$$

Here, $J\left(\boldsymbol{k}_{l}\right)$ denotes the Jakobian of $\boldsymbol{h}(\boldsymbol{k})$ at the singular point $\boldsymbol{k}_{l}$. The integral over the Berry curvature $\boldsymbol{\Omega}$ therefore equals $2 \pi$ times the Brouwer degree of the map $\boldsymbol{h}(\boldsymbol{k}) / h(\boldsymbol{k})$ from $T^{2}$ to $S^{2}$.

The mapping degree $C_{\text {Brouwer }}$ is a homotopy invariant of continuous maps between orientable manifolds [34]. For the simple case of maps from $S^{1}$ to $S^{1}$, it is just the winding number. In higher dimensions, and if base and image manifold are topologically the same, $C_{\text {Brouwer }}$ can be visualized by the number of "wrappings" associated with the map. Here, however, the situation is different because the maps go from $T^{2}$ to $S^{2}$ and even turn out to be singular, i.e. they are not continuous everywhere. Nevertheless, as we show in Sec. IV, a simple visualization is possible.

\section{B. Skyrmion number}

Equation (9) can be alternatively derived by directly evaluating $\boldsymbol{\Omega}$. With

$$
\partial_{1} v_{2}-\partial_{2} v_{1}=\Omega
$$

and the shorthand notation

$$
J_{a b}=\left(\partial_{1} h_{a}\right)\left(\partial_{2} h_{b}\right)-\left(\partial_{1} h_{b}\right)\left(\partial_{2} h_{a}\right)
$$

for $a, b \in\{x, y, z\}$, we find (see Appendix A)

$$
\Omega=\frac{1}{2} \frac{1}{h^{3}}\left[h_{x} J_{y z}+h_{y} J_{z x}+h_{z} J_{x y}\right],
$$

$\Omega(\boldsymbol{k})$ is therefore identified as a smooth function of $\boldsymbol{k}$ if $\boldsymbol{h}$ is smooth and vanishes nowhere, i.e. the divergences of $\boldsymbol{v}$ have been lifted in $\Omega$. We rewrite the Berry curvature also in the standard vectorial notation

$$
\Omega(\boldsymbol{k})=\frac{1}{2} \frac{\boldsymbol{h}}{h^{3}} \cdot\left[\partial_{1} \boldsymbol{h} \times \partial_{2} \boldsymbol{h}\right] .
$$

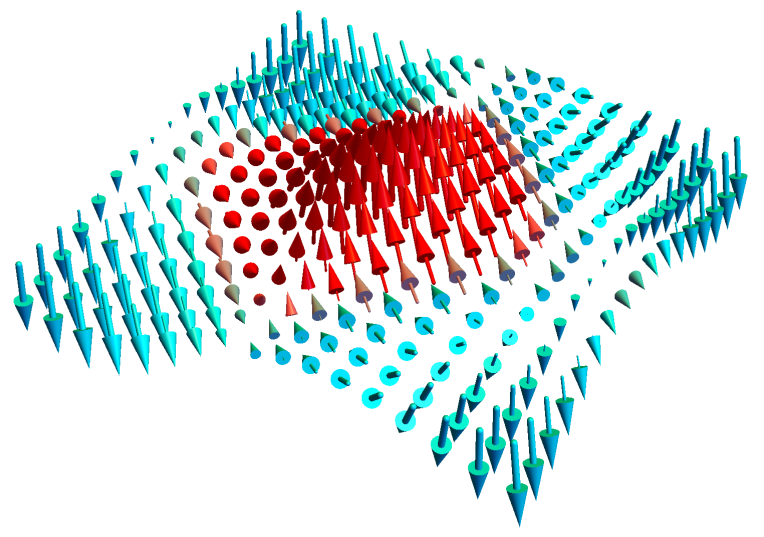

FIG. 1. (Color online) Skyrmion texture of the Bloch vector $\boldsymbol{h}(\boldsymbol{k})$ of a two-dimensional two-band topological insulator. Specifically, $\boldsymbol{h}(\boldsymbol{k})=\left(\alpha \sin k_{y},-\alpha \sin k_{x}, M+B\left[2-\cos k_{x}-\right.\right.$ $\left.\left.\cos k_{y}\right]\right)$ is plotted in the Brillouin zone of a square lattice for $\alpha=B=1$ and $M=-2[2,35]$.

Two times the Berry curvature is thus

$$
\Omega_{\mathrm{h}}(\boldsymbol{k})=\hat{\boldsymbol{h}} \cdot\left[\partial_{1} \hat{\boldsymbol{h}} \times \partial_{2} \hat{\boldsymbol{h}}\right],
$$

where we have introduced the normalized $\hat{\boldsymbol{h}}=\boldsymbol{h} / \boldsymbol{h}$ and used Eq. (A16) (see Appendix A). This $\Omega_{\mathrm{h}}$ is associated to another, seemingly different topological invariant, the skyrmion number. In fact, for a two-band topological insulator described by the Hamiltonian of Eq. (1) with a special choice of $\boldsymbol{h}(\boldsymbol{k})$, the Bloch-vector field over the BZ represents a skyrmion (see Fig. 1), and the skyrmion number is defined as [2, 29]

$$
N_{\mathrm{S}}=\frac{1}{4 \pi} \int_{B Z} \Omega_{\mathrm{h}}(\boldsymbol{k}) \mathrm{d}^{2} k .
$$

$N_{\mathrm{S}}$ is necessarily an integer and equals $C_{\text {Brouwer }}$, as shown in Appendix A.

\section{Spin texture}

The $\boldsymbol{k}$-dependent groundstate expectation value of the pseudospin

$$
\boldsymbol{S}(\boldsymbol{k})=\frac{1}{2}\left\langle c_{\boldsymbol{k} s}^{\dagger} \boldsymbol{\sigma}_{s s^{\prime}} c_{\boldsymbol{k} s^{\prime}}\right\rangle
$$

reads - obtained with the normalized eigenspinors $\boldsymbol{u}_{-}(\boldsymbol{k})$ in Eq. (2):

$$
\begin{aligned}
& S_{x}(\boldsymbol{k})=\operatorname{Re}\left[u_{1}^{*}(\boldsymbol{k}) u_{2}(\boldsymbol{k})\right]=\frac{h_{x}}{2 h}, \\
& S_{y}(\boldsymbol{k})=\operatorname{Im}\left[u_{1}^{*}(\boldsymbol{k}) u_{2}(\boldsymbol{k})\right]=\frac{h_{y}}{2 h}, \\
& S_{z}(\boldsymbol{k})=\frac{1}{2}\left[\left|u_{1}(\boldsymbol{k})\right|^{2}+\left|u_{2}(\boldsymbol{k})\right|^{2}\right]=\frac{h_{z}}{2 h} .
\end{aligned}
$$


Therefore, $\boldsymbol{S}(\boldsymbol{k})=\boldsymbol{h}(\boldsymbol{k}) / 2 h(\boldsymbol{k})$, and the topological invariant is equivalently computed using the spin expectation value. We write with the normalized $\hat{\boldsymbol{S}}=\boldsymbol{S} /|\boldsymbol{S}|$

$$
N_{\mathrm{S}}=\frac{1}{4 \pi} \int_{B Z} \hat{\boldsymbol{S}} \cdot\left[\partial_{1} \hat{\boldsymbol{S}} \times \partial_{2} \hat{\boldsymbol{S}}\right] \mathrm{d}^{2} k
$$

with the $\boldsymbol{k}$-dependence of $\hat{\boldsymbol{S}}(\boldsymbol{k})$ suppressed. Obviously, the Berry curvature can be expressed in terms of the expectation value of the spin and, correspondingly, we introduce

$$
\Omega_{\mathrm{S}}(\boldsymbol{k})=\hat{\boldsymbol{S}} \cdot\left[\partial_{1} \hat{\boldsymbol{S}} \times \partial_{2} \hat{\boldsymbol{S}}\right]
$$

As $\Omega_{\mathrm{S}}(\boldsymbol{k})=\Omega_{h}(\boldsymbol{k})$, the spin texture within the BZ is therefore, up to normalization, equivalent to the Bloch vector field displayed in Fig. 1.

Here we have already denoted $\boldsymbol{S}(\boldsymbol{k})$ as "spin" rather than pseudospin. Indeed, it is irrelevant whether this discrete degree of freedom is a pseudospin or the true electron spin, as long as we do not introduce additional interaction terms. In the spin language, the Bloch vector $\boldsymbol{h}(\boldsymbol{k})$ parametrizes the spin-orbit coupling and the Zeeman coupling to an external magnetic field. In the subsequent sections we will address a model superconductor that has spin degrees of freedom included naturally.

\section{Kubo formula}

Within linear response theory, the Hall conductance $\sigma_{x y}=\left(e^{2} / h\right) C$ is determined by the Kubo formula,

$$
\begin{aligned}
C=\frac{i}{2 \pi} \int_{B Z} \mathrm{~d}^{2} k & \sum_{n \neq m} \frac{f\left(E_{n \boldsymbol{k}}\right)-f\left(E_{m \boldsymbol{k}}\right)}{\left(E_{n \boldsymbol{k}}-E_{m \boldsymbol{k}}\right)^{2}} \\
& \times\left\langle n, \boldsymbol{k}\left|\hat{J}_{x}\right| m, \boldsymbol{k}\right\rangle\left\langle m, \boldsymbol{k}\left|\hat{J}_{y}\right| n, \boldsymbol{k}\right\rangle .
\end{aligned}
$$

The eigenenergies are labeled by $n$ (or $m$ ) and momentum $\boldsymbol{k} ; \hat{J}_{x}, \hat{J}_{y}$ are the components of the paramagnetic current operator (in units of $e / h$ ), and $f(E)$ denotes the Fermi function. In the zero temperature limit, $C=C_{\mathrm{TKNN}}$, the first Chern number. This is verified by explicitly evaluating the Kubo formula for the Hamiltonian Eq. (1) [35]. The Kubo formula Eq. (20) can be cast into the form:

$$
C_{\mathrm{TKNN}}=\frac{1}{2 \pi} \int_{B Z} \mathrm{~d}^{2} k \Omega_{\mathrm{B}}(\boldsymbol{k})
$$

where $\Omega_{\mathrm{B}}(\boldsymbol{k})$ is the Berry curvature [6]

$$
\Omega_{\mathrm{B}}(\boldsymbol{k})=i \sum_{n} \boldsymbol{\nabla}_{\boldsymbol{k}} \times\left.\left\langle n, \boldsymbol{k}\left|\boldsymbol{\nabla}_{\boldsymbol{k}}\right| n, \boldsymbol{k}\right\rangle\right|_{z}
$$

For the two-band model system we identify $| \pm, \boldsymbol{k}\rangle$ with $\boldsymbol{u}_{ \pm}(\boldsymbol{k})$ from Eq. (2) to arrive at Eq. (7).

To summarize, we identified $2 \Omega_{\mathrm{B}}(\boldsymbol{k})$ with the integrand of the skyrmion number integral, either in terms of the (a) $\boldsymbol{H}=\left(0,0, H_{z}\right)$

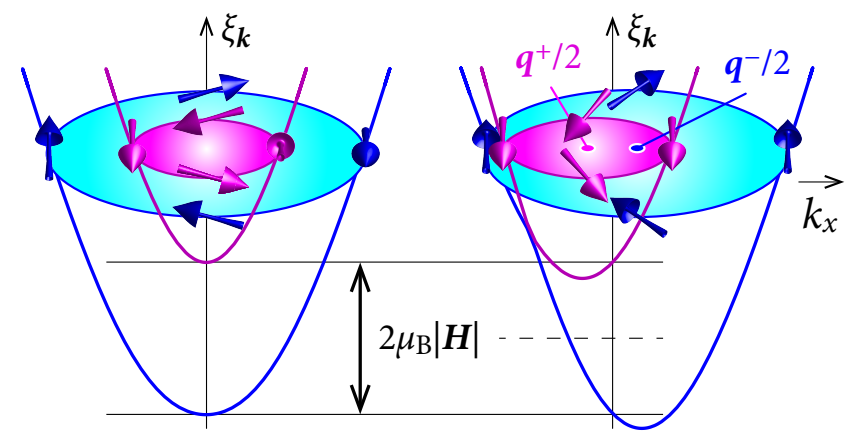

FIG. 2. (Color online) Band dispersions $\xi_{k}^{+}$(pink), $\xi_{\boldsymbol{k}}^{-}$(blue) with Rashba spin-orbit coupling and (a) out-of-plane and (b) in-plane Zeeman field. The centers of the shifted Fermi surfaces in (b) are at the momenta $\boldsymbol{q}^{+} / 2=\left(q^{+} / 2,0\right)$ and $\boldsymbol{q}^{-} / 2=\left(q^{-} / 2,0\right)$, respectively, with $q^{+} \sim q^{-}[37,42]$.

normalized Bloch vector $\Omega_{\mathrm{h}}(\boldsymbol{k})$ or in terms of the normalized spin vector $\Omega_{\mathrm{S}}(\boldsymbol{k})$

$$
2 \Omega(\boldsymbol{k})=\Omega_{\mathrm{h}}(\boldsymbol{k})=\Omega_{\mathrm{S}}(\boldsymbol{k})=2 \Omega_{\mathrm{B}}(\boldsymbol{k}) .
$$

The skyrmion number integral $N_{\mathrm{S}}$ is the Brouwer degree

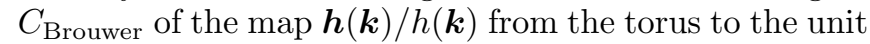
sphere

$$
\frac{1}{2 \pi} \int_{B Z} \boldsymbol{\Omega}=C_{\text {Brouwer }}=N_{\mathrm{S}}=C_{\mathrm{TKNN}},
$$

which equals the TKNN number $C_{\mathrm{TKNN}}$ of the transverse Hall conductance.

The topological insulator is distinct from the standard band insulators through finite, integer numbered topological invariants, an example of which is the integer $C_{\text {TKNN }}$. This is true for electronic systems with discrete translational invariance and, consequently, a well-defined BZ. The general mechanism to convert a band insulator into a topological insulator is band inversion; spin-orbit coupling is the underlying mechanism which inverts the usual ordering of conduction and valence bands [36]. In the example for a skyrmion texture of the Bloch vector in Fig. 1 band inversion is easily achieved by sign change to a negative parameter $M$ (see caption of Fig. 1) [35].

\section{S-WAVE SUPERCONDUCTOR WITH SPIN-ORBIT COUPLING}

We now apply these concepts and the established topological invariants to a superconductor. Specifically, we examine the topological character of a two-dimensional (2D) $s$-wave superconductor with Rashba SOC in an external magnetic field focussing on its spin texture in momentum space. The magnetic field is assumed to couple exclusively to the electron spin via the Zeeman energy 
and not to the orbital motion of the electrons. The reasoning for this ansatz is that the topological character of the superconductor is preserved upon rotating the Zeeman field from an orientation perpendicular to the superconducting plane towards an in-plane orientation [37]. For the latter orientation complications due to vortex physics and circulating supercurrents are absent.

For simplicity we use a one-band tight-binding model on a square lattice with periodic boundary conditions and nearest-neighbor hopping amplitude $t$. We start from a BCS pairing Hamiltonian for a superconducting state with $s$-wave symmetry and supplement it by $\mathcal{H}_{S}$ which contains Rashba SOC and the Zeeman energy. We follow the general notation of the previous chapter, and with

$$
\mathcal{H}_{\mathrm{S}}=\sum_{\boldsymbol{k} ; s, s^{\prime}= \pm 1}\left(\alpha \boldsymbol{g}(\boldsymbol{k})+\mu_{\mathrm{B}} \boldsymbol{H}\right) \cdot \boldsymbol{\sigma}_{s s^{\prime}} c_{\boldsymbol{k}, s}^{\dagger} c_{\boldsymbol{k}, s^{\prime}}
$$

the Bloch vector is now specified as $\boldsymbol{h}(\boldsymbol{k})=\alpha \boldsymbol{g}(\boldsymbol{k})+$ $\mu_{\mathrm{B}} \boldsymbol{H}$. Rashba SOC with strength $\alpha$ is represented by the vector $\boldsymbol{g}(\boldsymbol{k})=\left(\sin k_{y},-\sin k_{x}, 0\right), \boldsymbol{H}$ denotes the external magnetic field and $\mu_{\mathrm{B}}$ is the Bohr magneton. Diagonalizing $\mathcal{H}_{0}+\mathcal{H}_{S}$, where $\mathcal{H}_{0}=\sum_{\boldsymbol{k}, s} \epsilon_{\boldsymbol{k}} c_{\boldsymbol{k}, s}^{\dagger} c_{\boldsymbol{k}, s}$ is the kinetic energy of the hopping motion, leads to the two spin-split chiral energy bands $\xi_{\boldsymbol{k}}^{ \pm}=\epsilon_{\boldsymbol{k}} \pm|\alpha \boldsymbol{g}(\boldsymbol{k})+\boldsymbol{H}|$. The chemical potential $\mu$ is hereby combined with the dispersion as $\epsilon_{\boldsymbol{k}}=-2 t\left(\cos k_{x}+\cos k_{y}\right)-\mu$.

The two chiral bands $\xi_{\boldsymbol{k}}^{ \pm}$are depicted in Fig. 2. The electrons are supposed to move in the $x$ - $y$-plane and the dispersion is shown for the two qualitatively different cases of an out-of-plane magnetic field $\boldsymbol{H}=\left(0,0, H_{z}\right)$ and an in-plane magnetic field $\boldsymbol{H}=\left(0, H_{y}, 0\right)$. In the chiral bands the spin is either parallel or antiparallel to $\boldsymbol{h}(\boldsymbol{k})$ and has a component which rotates either counterclockwise or clockwise upon circulating the Fermi surfaces. For an in-plane magnetic field the centers of the Fermi surfaces for the $\xi_{\boldsymbol{k}}^{+}$and the $\xi_{\boldsymbol{k}}^{-}$bands shift in opposite directions away from the $\Gamma$ point and perpendicular to the magnetic field. For a finite in-plane field component the superconducting state will therefore necessarily involve Cooper pairs with finite center-of-mass momenta (COMM) [37, 49-54].

Allowing for an arbitrary magnetic field orientation the ansatz for the superconducting state has to include the option to form electron pairs with finite COMM. Assuming a local, on-site pairing interaction, the superconducting order parameter is calculated self-consistently from $\mathcal{H}=\mathcal{H}_{0}+\mathcal{H}_{S}+\mathcal{H}_{I}$ with [45]

$$
\mathcal{H}_{\mathrm{I}}=\sum_{\boldsymbol{k}, \boldsymbol{q}} \sum_{s} s\left[\Delta_{\boldsymbol{q}}^{*} c_{-\boldsymbol{k}+\boldsymbol{q},-s} c_{\boldsymbol{k}, s}+\Delta_{\boldsymbol{q}} c_{\boldsymbol{k}, s}^{\dagger} c_{-\boldsymbol{k}+\boldsymbol{q},-s}^{\dagger}\right]
$$

where the summation is performed over all possible COMMs $\boldsymbol{q}$ of the electron pairs. The singlet order parameter for COMM $\boldsymbol{q}$ is calculated as

$$
\Delta_{\boldsymbol{q}}=-\frac{V}{2 N} \sum_{\boldsymbol{k}^{\prime}}\left\langle c_{-\boldsymbol{k}^{\prime}+\boldsymbol{q}, \downarrow} c_{\boldsymbol{k}^{\prime}, \uparrow}-c_{-\boldsymbol{k}^{\prime}+\boldsymbol{q}, \uparrow} c_{\boldsymbol{k}^{\prime}, \downarrow}\right\rangle,
$$

where $V$ is the pairing-interaction strength.

If all electron pairs carry the same COMM $\boldsymbol{q}, \mathcal{H}=$ $\sum_{\boldsymbol{k}} \mathbf{C}_{\boldsymbol{k}}^{\dagger} \mathcal{H}(\boldsymbol{k}) \mathbf{C}_{\boldsymbol{k}}$ is represented by the $4 \times 4$ matrix

$$
\mathcal{H}(\boldsymbol{k})=\left(\begin{array}{cc}
\epsilon_{\boldsymbol{k}} \sigma^{0}+\boldsymbol{h}(\boldsymbol{k}) \cdot \boldsymbol{\sigma} & i \sigma^{y} \Delta_{\boldsymbol{q}} \\
-i \sigma^{y} \Delta_{\boldsymbol{q}}^{*} & -\epsilon_{-\boldsymbol{k}+\boldsymbol{q}} \sigma^{0}-\boldsymbol{h}(-\boldsymbol{k}+\boldsymbol{q}) \cdot \boldsymbol{\sigma}^{*}
\end{array}\right)
$$

with $\mathbf{C}_{\boldsymbol{k}}^{\top}=\left(c_{\boldsymbol{k}, \uparrow}, c_{\boldsymbol{k}, \downarrow}, c_{-\boldsymbol{k}+\boldsymbol{q}, \uparrow}^{\dagger}, c_{-\boldsymbol{k}+\boldsymbol{q}, \downarrow}^{\dagger}\right)$.

It is instructive to rewrite the Hamiltonian in the helicity basis, i.e. in terms of the quasiparticle operators $a_{\boldsymbol{k}, \pm}$ which diagonalize $\mathcal{H}_{0}+\mathcal{H}_{S}$ [38]. These operators generate the band eigenstates with energies $\xi_{\boldsymbol{k}}^{ \pm}$and are obtained by the transformation

$$
\left(\begin{array}{l}
a_{\boldsymbol{k},+} \\
a_{\boldsymbol{k},-}
\end{array}\right)=\frac{1}{\sqrt{2 h(\boldsymbol{k})}}\left(\begin{array}{cc}
\sqrt{h(\boldsymbol{k})+h_{z}(\boldsymbol{k})} & \phi_{\boldsymbol{k}}^{+} \\
-\sqrt{h(\boldsymbol{k})-h_{z}(\boldsymbol{k})} & \phi_{\boldsymbol{k}}^{-}
\end{array}\right)\left(\begin{array}{l}
c_{\boldsymbol{k}, \uparrow} \\
c_{\boldsymbol{k}, \downarrow}
\end{array}\right)
$$

with $h(\boldsymbol{k})=|\boldsymbol{h}(\boldsymbol{k})|$ and

$$
\phi_{\boldsymbol{k}}^{ \pm}=\frac{h_{x}(\boldsymbol{k})-i h_{y}(\boldsymbol{k})}{\sqrt{h(\boldsymbol{k}) \pm h_{z}(\boldsymbol{k})}} .
$$

In the helicity basis, for a single COMM $\boldsymbol{q}$, the Hamiltonian takes the form $\mathcal{H}=\sum_{\boldsymbol{k}} \mathcal{A}_{\boldsymbol{k}}^{\dagger} \mathcal{H}^{\mathrm{hb}}(\boldsymbol{k}) \mathcal{A}_{\boldsymbol{k}}$ with $\mathcal{A}_{\boldsymbol{k}}^{\top}=$ $\left(a_{\boldsymbol{k},+}, a_{-\boldsymbol{k}+\boldsymbol{q},+}^{\dagger}, a_{\boldsymbol{k},-}, a_{-\boldsymbol{k}+\boldsymbol{q},-}^{\dagger}\right)$ and the $4 \times 4$ matrix

$$
\mathcal{H}^{\mathrm{hb}}(\boldsymbol{k})=\left(\begin{array}{cccc}
\epsilon_{\boldsymbol{k}}+h(\boldsymbol{k}) & \Delta^{++}(\boldsymbol{k}, \boldsymbol{q}) & 0 & \Delta^{+-}(\boldsymbol{k}, \boldsymbol{q}) \\
\Delta^{++^{*}}(\boldsymbol{k}, \boldsymbol{q}) & -\epsilon_{-\boldsymbol{k}+\boldsymbol{q}}-h(-\boldsymbol{k}+\boldsymbol{q}) & \Delta^{-+}(\boldsymbol{k}, \boldsymbol{q}) & 0 \\
0 & \Delta^{-+^{*}}(\boldsymbol{k}, \boldsymbol{q}) & \epsilon_{\boldsymbol{k}}-h(\boldsymbol{k}) & \Delta^{--}(\boldsymbol{k}, \boldsymbol{q}) \\
\Delta^{+-^{*}}(\boldsymbol{k}, \boldsymbol{q}) & 0 & \Delta^{--^{*}}(\boldsymbol{k}, \boldsymbol{q}) & -\epsilon_{-\boldsymbol{k}+\boldsymbol{q}}+h(-\boldsymbol{k}+\boldsymbol{q})
\end{array}\right)
$$

$\Delta^{+-}(\boldsymbol{k}, \boldsymbol{q})$ and $\boldsymbol{\Delta}^{-+}(\boldsymbol{k}, \boldsymbol{q})$ denote the inter-band pairing amplitudes. The intra-band pairing amplitudes $\Delta^{++}(\boldsymbol{k}, \boldsymbol{q})$ and $\Delta^{--}(\boldsymbol{k}, \boldsymbol{q})$ are odd functions with respect to interchanging the momenta $\boldsymbol{k}$ and $\boldsymbol{-} \boldsymbol{k}+\boldsymbol{q}$ [42], as can be explicitly verified from their functional form given in
Appendix B. The matrix elements on the skew diagonal in Eq. (31) lead to structures in the density of states away from the Fermi energy. Hence, they do not affect the closing of the bulk energy gap and an eventual topological phase transition [46]. 
For the special case $H_{x}=H_{y}=0$, i.e. for an out-ofplane orientation of the magnetic field, intra-band pairing does not require a finite COMM, and for $\boldsymbol{q}=\mathbf{0}$ the pairing amplitude simply reduces to

$$
\begin{aligned}
\Delta^{++}(\boldsymbol{k}, \mathbf{0}) & =-\frac{\Delta_{\mathbf{0}}}{h(\boldsymbol{q})}\left(h_{x}(\boldsymbol{k})-i h_{y}(\boldsymbol{k})\right) \\
& =-g \frac{\Delta_{\mathbf{0}}}{h(\boldsymbol{k})}\left(\sin k_{y}+i \sin k_{x}\right),
\end{aligned}
$$

which is evidently odd in $\boldsymbol{k}$. In fact, the pairing state in each of the helical bands acquires the form of a spinless $p_{y}+i p_{x}$ superconductor, similar to the proximity induced superconductivity on the surface of a topological insulator in contact with an $s$-wave superconductor [39] or in a semiconductor quantum well coupled to an $s$-wave superconductor and a ferromagnetic insulator $[9,11]$. The order parameters on the two Fermi surface sheets are equal in magnitude, but since

$$
\Delta^{--}(\boldsymbol{k}, \mathbf{0})=g \frac{\Delta_{\mathbf{0}}}{h(\boldsymbol{k})}\left(\sin k_{y}-i \sin k_{x}\right),
$$

they have the opposite chirality [9]. Beyond a critical magnetic field strength $H_{\mathrm{t}}$, the upper $\xi_{\boldsymbol{k}}^{+}$band is unoccupied; $\Delta^{++}$and the inter-band pairing amplitudes then necessarily vanish. The pairing on the remaining lower $\xi_{\boldsymbol{k}}^{-}$band has a unique chirality. This is the origin for a topologically non-trivial character of the superconducting state for $H>H_{\mathrm{t}}$.

\section{MOMENTUM-SPACE SPIN TEXTURES}

The superconducting state is represented by the $4 \times 4$ matrix of Eq. (28) in Nambu space. The four eigenvectors $\boldsymbol{u}_{n}(\boldsymbol{k})$ describe the specific mixture of electrons and holes in each eigenstate for a given momentum $\boldsymbol{k}$. In the general situation with SOC and an arbitrarily oriented magnetic $\mathcal{H}(\boldsymbol{k})$ cannot be written in a block-diagonal form due to the interband pairing amplitudes, as is evident from the structure of the Hamiltonian matrix $\mathcal{H}^{\mathrm{hb}}(\boldsymbol{k})$ (see Eq. (31)) in the helicity basis. In this general case, the eigenvectors $\boldsymbol{u}_{n}(\boldsymbol{k})$ are therefore calculated numerically which thereby requires to determine the optimum COMM $\boldsymbol{q}$. The eigenvectors are subsequently used to evaluate the spin expectation values $\boldsymbol{S}(\boldsymbol{k})$ in the superconducting state. Their texture in momentum space is analyzed separately for an out-of-plane orientation of the magnetic field and for a mixed situation, in which the magnetic field has both out-of-plane and in-plane components.

\section{A. Out-of-plane magnetic field, $T=0$}

We start with a magnetic field $\boldsymbol{H}=\left(0,0, H_{z}\right)$, for which the two Fermi surface sheets are both centered at the $\Gamma$-point and intra-band electron pairs form with
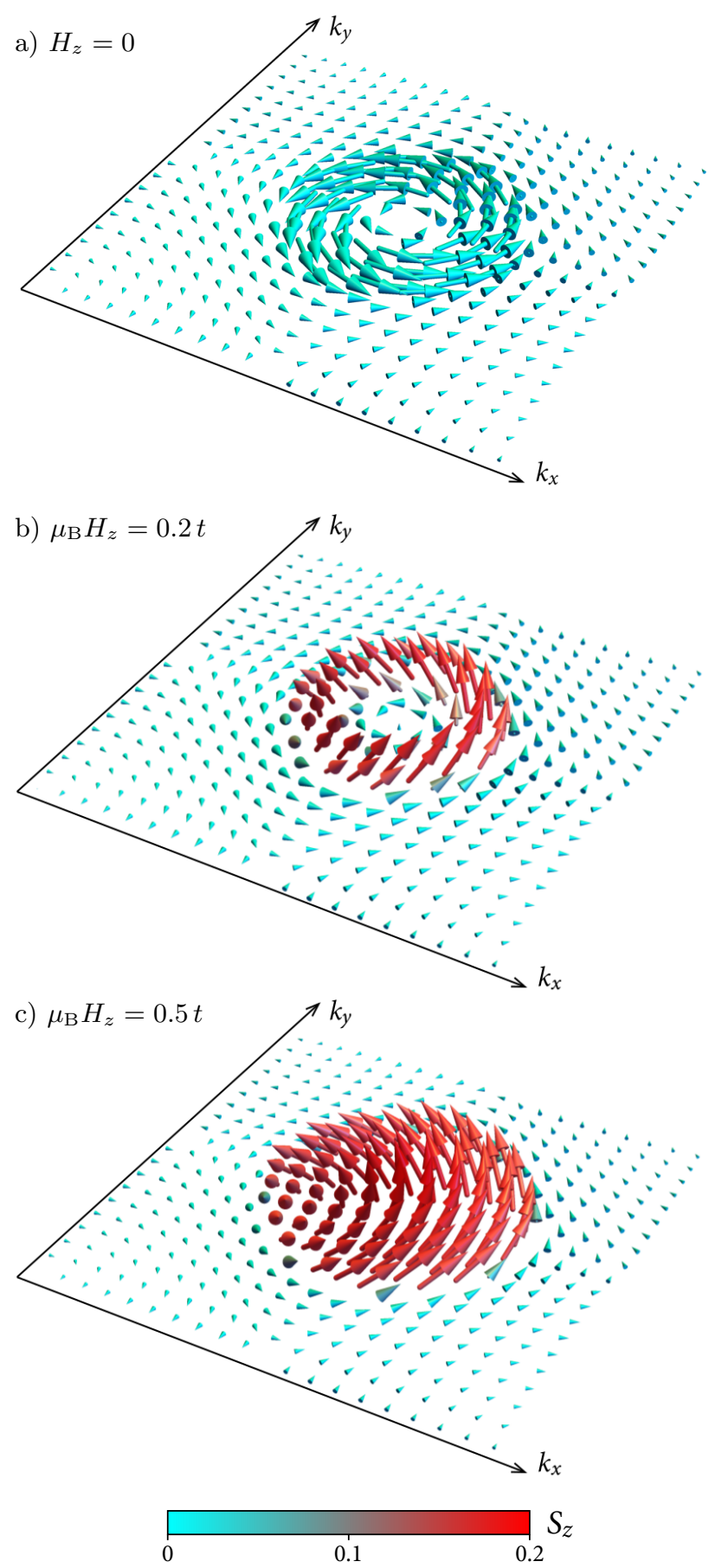

FIG. 3. (Color online) Ground-state spin-expectation value $\boldsymbol{S}(\boldsymbol{k})$ in the first Brillouin zone, $k_{x}, k_{y} \in[-\pi, \pi]$, for an outof-plane orientation of the magnetic field, i.e. $H_{x}=H_{y}=0$. The further parameters are: $\Delta=0.15 t, \alpha=0.4 t$, and $\mu=-3.2 t$. The critical Zeeman field for these parameters is $\mu_{\mathrm{B}} H_{\mathrm{t}} \sim 0.4 t$. The color of each spin represents the value of the out-of-plane component $S_{z}(\boldsymbol{k})$; its magnitude is determined from the color bar at the bottom. The skyrmion counting number is $N_{\mathrm{S}}=0$ in (a) and (b), while $N_{\mathrm{S}}=1 / 2$ in (c). 
zero COMM. In this comparatively simple situation we refrain from a self-consistent calculation and assume a finite fixed order parameter $\Delta \equiv \Delta_{\boldsymbol{q}=\mathbf{0}}$. Figure 3 illustrates the spin texture in the first BZ for three different field strengths, including $H_{z}=0$. These results were obtained at zero temperature for the parameter set given in the caption. Figures $3 \mathrm{a}$ and $3 \mathrm{~b}$ depict the texture in the topologically trivial superconducting state. For $H_{z}=0$, there is no net spin magnetization, and $\boldsymbol{S}(\boldsymbol{k})$ is confined to the $x-y$-plane. The spin winding reflects the spin-momentum locking due to SOC. For the finite Zeeman field in Fig. 3b, imprints of the two $\Gamma$-point centered Fermi surfaces with unequal areas are visible, and the electrons as a whole have picked up a finite spin magnetization. For the larger Zeeman field in Fig. 3c, $H_{z}$ has exceeded its critical value $H_{\mathrm{t}}=\sqrt{\Delta^{2}+\epsilon_{\mathbf{0}}^{2}}$ [8]. For $H_{z}>H_{\mathrm{t}}$, all electrons are in the lower $\xi_{\boldsymbol{k}}^{-}$band and form a topological superconductor.

The topological transition is captured by the changes in the spin texture $[28,47]$. In order to verify that the spin texture indeed signifies the transition, we calculate the skyrmion number as the corresponding topological invariant. For this purpose, we can utilize the normalized spin-vector expectation value $\hat{\boldsymbol{S}}(\boldsymbol{k})=\boldsymbol{S}(\boldsymbol{k}) /|\boldsymbol{S}(\boldsymbol{k})|$ for the required BZ integral, as outlined in chapter II. With the spin product

$$
\Omega_{\mathrm{S}}(\boldsymbol{k})=\hat{\boldsymbol{S}}(\boldsymbol{k}) \cdot\left(\partial_{k_{x}} \hat{\boldsymbol{S}}(\boldsymbol{k}) \times \partial_{k_{y}} \hat{\boldsymbol{S}}(\boldsymbol{k})\right)
$$

the skyrmion number of the spin texture is

$$
N_{\mathrm{S}}=\frac{1}{4 \pi} \int_{B Z} \Omega_{\mathrm{S}}(\boldsymbol{k}) \mathrm{d}^{2} k .
$$

$N_{\mathrm{S}}$ takes integer values if the map $\hat{\boldsymbol{S}}(\boldsymbol{k})$ from the BZ to the unit sphere is surjective and everywhere continuous.

We now introduce a different normalization of the expectation value of the spin, $\boldsymbol{S}_{\mathrm{L}}(\boldsymbol{k})$, in which $S_{x}$ and $S_{y}$ are normalized only to the in-plane components

$$
\boldsymbol{S}_{\mathrm{L}}(\boldsymbol{k})=\left(\frac{S_{x}}{\sqrt{S_{x}^{2}+S_{y}^{2}}}, \frac{S_{y}}{\sqrt{S_{x}^{2}+S_{y}^{2}}}, S_{z}\right)
$$

and we define

$$
\Omega_{\mathrm{L}}(\boldsymbol{k})=\boldsymbol{S}_{\mathrm{L}}(\boldsymbol{k}) \cdot\left(\partial_{k_{x}} \boldsymbol{S}_{\mathrm{L}}(\boldsymbol{k}) \times \partial_{k_{y}} \boldsymbol{S}_{\mathrm{L}}(\boldsymbol{k})\right)
$$

This spin product has the remarkable property to be pointwise identical to the Berry curvature:

$$
\Omega_{\mathrm{L}}(\boldsymbol{k})=\Omega_{\mathrm{B}}(\boldsymbol{k})
$$

for each momentum $\boldsymbol{k}$. Heretofore, this equality could be confirmed only numerically. The zero-temperature quantity $\Omega_{\mathrm{L}}$ will prove useful when we extend its range of validity to finite temperatures in Sec. IV B.

In the absence of an analytical proof of the equality (38) we resort to the relations established for the two-band model in Sec. A: In Eq. (A21) we introduced a similar spin quantity, $\overline{\boldsymbol{S}}_{1}(\boldsymbol{k})$, where the particular normalization of $\overline{\boldsymbol{S}}_{1}(\boldsymbol{k})$ describes the mapping of the BZ to the barrel of a cylinder with unit radius - while that of $\hat{\boldsymbol{S}}(\boldsymbol{k})$ involves the projection onto the unit sphere. Only $\hat{\boldsymbol{S}}(\boldsymbol{k})$ and $\overline{\boldsymbol{S}}_{1}(\boldsymbol{k})$ are proven to generate the topological invariant $N_{\mathrm{S}}$. Nevertheless, it is striking that Eq. (38) holds. Moreover, $2 N_{\mathrm{S}}$ of Eq. (35) is identical to the Chern (or TKNN number) given in Eq. (21)

$$
2 N_{\mathrm{S}}=\frac{1}{2 \pi} \int_{B Z} \Omega_{\mathrm{B}}(\boldsymbol{k}) \mathrm{d}^{2} k
$$

in the present case. While the relation between the skyrmion number of the spin texture as given in Eq. (35) and the Berry curvature is a numerical finding, the spin texture may be analyzed analytically in its own right, as is done in the following.

For the spin textures in Figs. 3a and $3 \mathrm{~b}$ with the Zeeman fields $H_{z}<H_{\mathrm{t}}$ the BZ integrals lead to $N_{\mathrm{S}}=0$. Instead, for the topological superconductor with the Zeeman field $H_{z}>H_{\mathrm{t}}$ as in Fig. 3c, we obtain $N_{\mathrm{S}}=1 / 2$. This latter non-trivial result may appear surprising at first sight, because $N_{\mathrm{S}}$ must be always zero, if the spin texture is a continuous map from the BZ to the unit sphere $S^{2}$. Here, in fact this map can never cover the entire sphere because $S_{z}$ attains only non-negative values for $H_{z} \geq 0$. However, the topological superconductor the spin texture corresponds to a very special singular map from the BZ to the upper half-sphere, which preserves a topological signature, as shown below.

Figure 3 illustrates that the components of $\boldsymbol{S}(\boldsymbol{k})$ become very small at the BZ boundary. In fact, $\boldsymbol{S}(\boldsymbol{k})$ vanishes at eight (in part equivalent) BZ boundary points $\boldsymbol{k}_{c}$, namely at the BZ corners $( \pm \pi, \pm \pi)$ and at the four points $( \pm \pi, 0)$ and $(0, \pm \pi)$ on the BZ faces. While also the normalized component $S_{z}(\boldsymbol{k}) /|\boldsymbol{S}(\boldsymbol{k})|$ vanishes at these $\boldsymbol{k}_{c}$, either $\lim _{\boldsymbol{k} \rightarrow \boldsymbol{k}_{c}} S_{x}(\boldsymbol{k}) /|\boldsymbol{S}(\boldsymbol{k})|$ or $\lim _{\boldsymbol{k} \rightarrow \boldsymbol{k}_{c}} S_{y}(\boldsymbol{k}) /|\boldsymbol{S}(\boldsymbol{k})|$ remain finite. The normalized vector field $\hat{\boldsymbol{S}}(\boldsymbol{k})$ can be defined at all points $\boldsymbol{k} \neq \boldsymbol{k}_{c}$ and the integrand in Eq. (35) is therefore well defined in the BZ, away from the momenta $\boldsymbol{k}_{c}$ on the BZ boundary. Considering the BZ as a chart of the torus $T^{2}$, the eight points $\boldsymbol{k}_{c}$ correspond to three points on the torus: $( \pm \pi, \pm \pi)$ are equivalent as well as $( \pm \pi, 0)$ and $(0, \pm \pi)$. For $H_{z}<H_{\mathrm{t}}$, another point with the same properties appears at the center of the BZ, $\boldsymbol{k}_{c}=\mathbf{0}$.

Close to these three (respectively four) points on the torus, the spin texture exhibits "vortex-like" characteristics. Due to such micro-vortices in momentumspace, the vector field $\hat{\boldsymbol{S}}$ is discontinuous at the momenta $\boldsymbol{k}_{c}$ (and also at $\boldsymbol{k}_{c}=\mathbf{0}$ for $H_{z}<H_{\mathrm{t}}$ ) because the image of each $\boldsymbol{k}_{c}$ is the full equator $\left(S_{z}=0\right)$ of the unit sphere $S^{2}$. More precisely, an infinitesimally small circle $\boldsymbol{k}_{c}+\varepsilon\left(\cos \phi \boldsymbol{e}_{1}+\sin \phi \boldsymbol{e}_{2}\right)$, with $\varepsilon \ll 1$ and two orthogonal unit vectors $\boldsymbol{e}_{1}$ and $\boldsymbol{e}_{2}$, is mapped onto $\left(\sqrt{1-\tilde{\varepsilon}^{2}} \cos \theta(\phi), \sqrt{1-\tilde{\varepsilon}^{2}} \sin \theta(\phi), \tilde{\varepsilon}(\phi)\right)$, where $\theta(\phi)$ and $\tilde{\varepsilon}(\phi)$ are functions of $\phi$ and $\tilde{\varepsilon}(\phi)$ is of the order $\varepsilon$. As the map $T^{2} \rightarrow S^{2}$ furnished by $\hat{\boldsymbol{S}}(\boldsymbol{k})$ is not continuous at the 


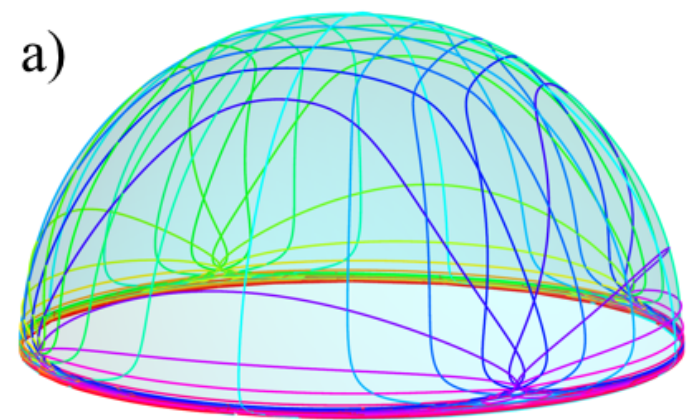

c)

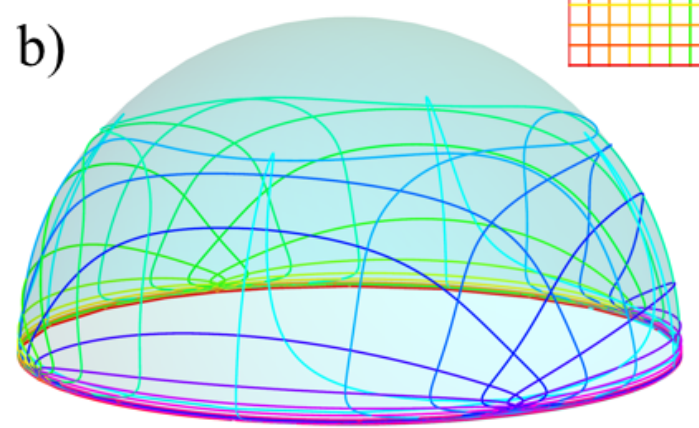

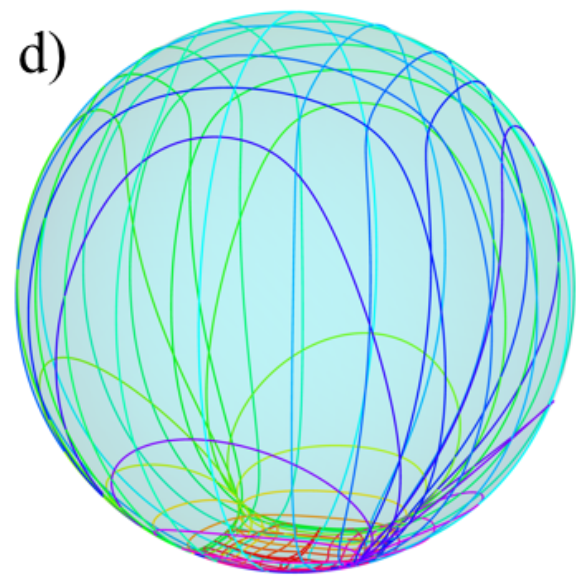

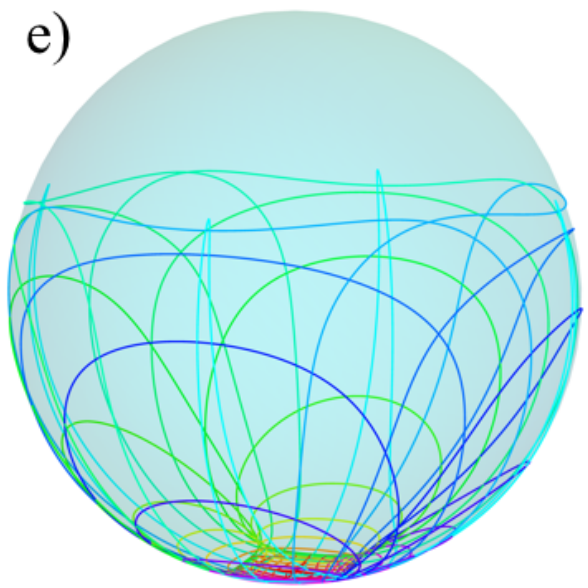

FIG. 4. (Color online) The maps $\hat{\boldsymbol{S}}(\boldsymbol{k})\left[(\mathrm{a})\right.$ and (b)] and $\hat{\boldsymbol{S}}_{\mathrm{reg}}(\boldsymbol{k})[(\mathrm{d})$ and (e)] for the topologically non-trivial [(a) and (d)] and trivial cases [(b) and (e)]. The colored rectangular grid in the BZ, depicted in (c), is mapped onto the corresponding lines on the (hemi-)spheres. Note the approximate restoration of the rectangular grid structure in the vicinity of the "south pole" in (d) and (e). For $N_{\mathrm{S}}^{(\text {meron })}=1[(\mathrm{a})$ and (d)] an almost regular pattern is also visible close to the "north pole". The distortion of the flat metric in the BZ is only weak in these areas. For these maps, the lattice size is $1600 \times 1600$, the chemical potential is $\mu=-3.6 t$ (which corresponds to a density $n=0.075$ ), the Rashba SOC strength is $\alpha=0.6 t$, and the gap is $\Delta=0.15 t$. The magnetic field is $\mu_{B} H_{z}=0.4 t$ for the trivial cases [(b) and (e)] and $\mu_{B} H_{z}=t$ for the topologically non-trival cases [(a) and (d)].

isolated points $\boldsymbol{k}_{c}$, no topologically meaningful quantity appears to be associated with it and the evaluation of Eq. (35) may be expected to yield arbitrary values.

Yet, the skyrmion counting number $N_{\mathrm{S}}$ is quantized, albeit not in integers but in half-integers. This apparent puzzle is resolved by the following observation: $S_{z}$ is confined to values $\geq 0$ for $H_{z} \geq 0$, signifying that the BZ is mapped into the upper hemisphere $S_{u}^{2}$. This manifold has a boundary $A$ (the equator) and is topologically equivalent to the disk $D^{2}$. The map given by $\hat{\boldsymbol{S}}(\boldsymbol{k})$ is continuous from $\left\{\boldsymbol{k} \in \mathrm{BZ}, \boldsymbol{k} \neq \boldsymbol{k}_{c}\right\}$ to the open hemisphere $S_{u}^{2} \backslash A$. Because the equator is the unique limit set of the images of any sequence of points converging towards one of the isolated points $\boldsymbol{k}_{c}$, we can construct from $\hat{\boldsymbol{S}}$ a continuous map $\hat{\boldsymbol{S}}_{\text {reg }}=\boldsymbol{\Phi} \circ \hat{\boldsymbol{S}}$ of the torus onto $S^{2}$, a compact manifold without boundary, by compactifying the open hemisphere $S_{u}^{2} \backslash A$ to a sphere via the function $\boldsymbol{\Phi}$, with

$$
\boldsymbol{\Phi}(x, y, z)=\left(2 x \sqrt{\frac{z}{z+1}}, 2 y \sqrt{\frac{z}{z+1}}, 2 z-1\right) .
$$

Here $\boldsymbol{\Phi}$ maps all points located on the equator of $S_{u}^{2}$ to the south pole $(0,0,-1)$ of $S^{2}$. In turn, $\hat{\boldsymbol{S}}_{\text {reg }}(\boldsymbol{k})$ maps the singular points $\boldsymbol{k}_{c}$ to $(0,0,-1)$, a neighborhood of any $\boldsymbol{k}_{c}$ to a neighborhood of the south pole, and $\hat{\boldsymbol{S}}_{\text {reg }}(\boldsymbol{k})$ is therefore continuous. The application of $\boldsymbol{\Phi}$ multiplies the surface element of $S^{2}$ by 2 (compare Eq. (A18)). Consequently, we define the quantity

$$
N_{\mathrm{S}}^{(\text {meron })}=\frac{1}{2 \pi} \int_{B Z} \Omega_{\mathrm{S}}(\boldsymbol{k}) \mathrm{d}^{2} k=\frac{1}{4 \pi} \int_{B Z} \Omega_{\mathrm{reg}}(\boldsymbol{k}) \mathrm{d}^{2} k .
$$


which always takes integer values. In analogy to the relations (34) and (37) we have introduced the spin product

$$
\Omega_{\mathrm{reg}}(\boldsymbol{k})=\hat{\boldsymbol{S}}_{\mathrm{reg}}(\boldsymbol{k}) \cdot\left(\partial_{k_{x}} \hat{\boldsymbol{S}}_{\mathrm{reg}}(\boldsymbol{k}) \times \partial_{k_{y}} \hat{\boldsymbol{S}}_{\mathrm{reg}}(\boldsymbol{k})\right) .
$$

We conclude that $N_{\mathrm{S}}$ is quantized in half-integers, and we associate the momentum-space spin structures in Fig. 3 with merons (see, e.g. Ref. 29), and $N_{\mathrm{S}}^{(\text {meron) }}$ is their counting number.

We can now characterize the spin textures for $H_{z}$ above and below $H_{\mathrm{t}}$ topologically, using the mapping degree of $\hat{\boldsymbol{S}}_{\text {reg }}$ in both cases. For $H_{z}>H_{\mathrm{t}}$, the complete upper hemisphere is covered by $\hat{\boldsymbol{S}}$ (see Fig. $4 \mathrm{a}$ and also Fig. 5a below). The three points $\boldsymbol{k}_{c}$ are mapped onto the equator and the northpole $(0,0,1)$ has a unique preimage. The regularized $\hat{\boldsymbol{S}}_{\text {reg }}$ maps the BZ to the full sphere, the south pole of which is a regular point with three preimages. This is the characteristics of Fig. 4d. As the number of preimages of all points of $S^{2}$ is odd, the map must be topologically non-trivial. Indeed, the Brouwer degree sums to 1 and therefore $N_{S}^{(\text {meron })}=1$.

The map $\hat{\boldsymbol{S}}$ in the topologically trivial case $H_{z}<H_{\mathrm{t}}$ is characterized by four preimages of the equator (respectively the south pole for $\hat{\boldsymbol{S}}_{\text {reg }}$ ). In addition to the three points $\boldsymbol{k}_{c}$ on the BZ boundary, the center of the $\mathrm{BZ}, \boldsymbol{k}_{c}=\mathbf{0}$, is also mapped to the equator while the area around the north pole $(0,0,1)$ is not covered by the maps

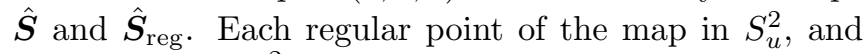
respectively in $S^{2}$, has an even number of preimages in the BZ, 0,2 or 4 , and the Brouwer degree sums to 0 . The singular map $\hat{\boldsymbol{S}}$ is shown in Fig. 4 b and the regular map $\hat{\boldsymbol{S}}_{\text {reg }}$ in Fig. 4 e.

Figures 5a and $5 \mathrm{c}$ display the images of some selected paths in the BZ (shown in Fig. 5b) onto $S_{u}^{2}$ by using the vertical projection of $S_{u}^{2}$ onto the unit disk in the $x-y$ plane. The case $H_{z}>H_{\mathrm{t}}$ is represented in Fig. 5a. The black circles around the center of the $\mathrm{BZ}$ are mapped to a neighborhood of the north pole, while the images of the areas around the singular points $\boldsymbol{k}_{c}$, at the BZ face centers and corners (blue/red/cyan circles), are located very close to the equator.

On the other hand, for $H_{z}<H_{\mathrm{t}}$ and $N_{\mathrm{S}}^{(\text {meron })}=0$ displayed in Fig. $5 \mathrm{c}$, the central area of the $\mathrm{BZ}$ is mapped into the vicinity of the equator as well, while the center of the disk (the north pole) is never reached. We infer from the lines traversing the BZ (orange, magenta, brown and green), that the torus is "folded back" before reaching the north pole and does not wrap around the hemisphere. Note that for both cases, $H_{z}>H_{\mathrm{t}}$ and $H_{z}<H_{\mathrm{t}}$, the symmetric paths, the diagonal (orange) line and the horizontal (magenta) line in the BZ are mapped to straight lines in the projection of the hemisphere.

These results allow to understand the topological difference between both types of maps (see Figs. $4 \mathrm{~d}$ and $4 \mathrm{e}$ ) in an intuitive way. We can construct a map $\mathcal{M}$ from $T^{2}$ to $S^{2}$ as follows: Consider a unit sphere together with a torus as subsets of $\mathbb{R}^{3}$ (see Fig. 6). The rays $\lambda \boldsymbol{v}$ from the

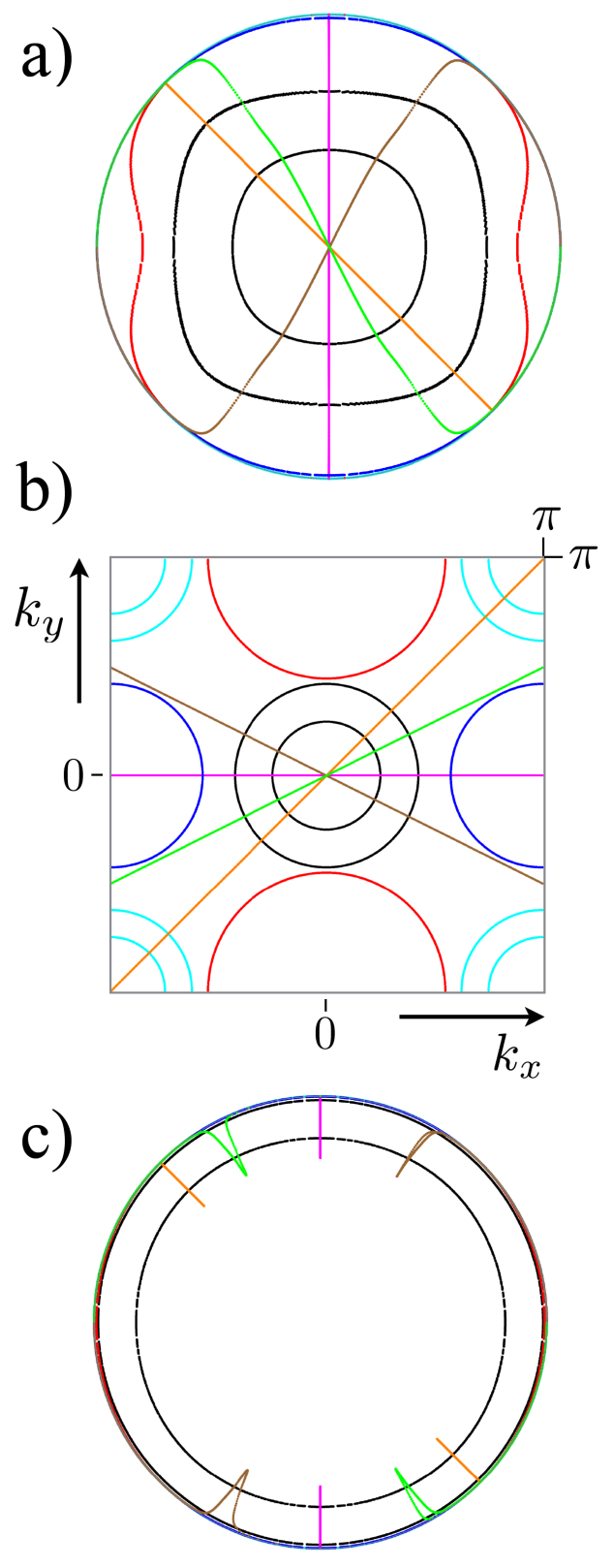

FIG. 5. (Color online) Images of selected paths in the BZ as obtained from the map $\hat{\boldsymbol{S}}(\boldsymbol{k})$; the paths are shown in panel (b). The topologically non-trivial case for $H_{z}>H_{\mathrm{t}}$ is presented in (a) and the topologically trivial case in (c). The image points in the upper hemisphere are mapped by vertical projection $[(x, y, z) \rightarrow(x, y, 0)]$ onto the unit disk. $S_{u}^{2}$ is completely covered for $H_{z}>H_{\mathrm{t}}$ while for $H_{z}<H_{\mathrm{t}}$ a part of $S_{u}^{2}$ is covered twice, and the overall coverage is incomplete. The parameter sets are the same as in Fig. 4.

center of the sphere - with a unit vector $\boldsymbol{v}$ and $\lambda>1-$ intersect the torus at points $\lambda_{1}[\boldsymbol{v}] \boldsymbol{v}, \ldots, \lambda_{j}[\boldsymbol{v}] \boldsymbol{v}$. Then we define the map $\mathcal{M}$ from the torus to $S^{2}$ by $\mathcal{M}\left(\lambda_{l}[\boldsymbol{v}] \boldsymbol{v}\right)=\boldsymbol{v}$ for all $l$. In Figs. 6a, $6 \mathrm{~b}$ and $6 \mathrm{c}$ we consider a ray through the south pole of the sphere (white point) which intersects the torus either three times (Figs. 6a and 6c) or 

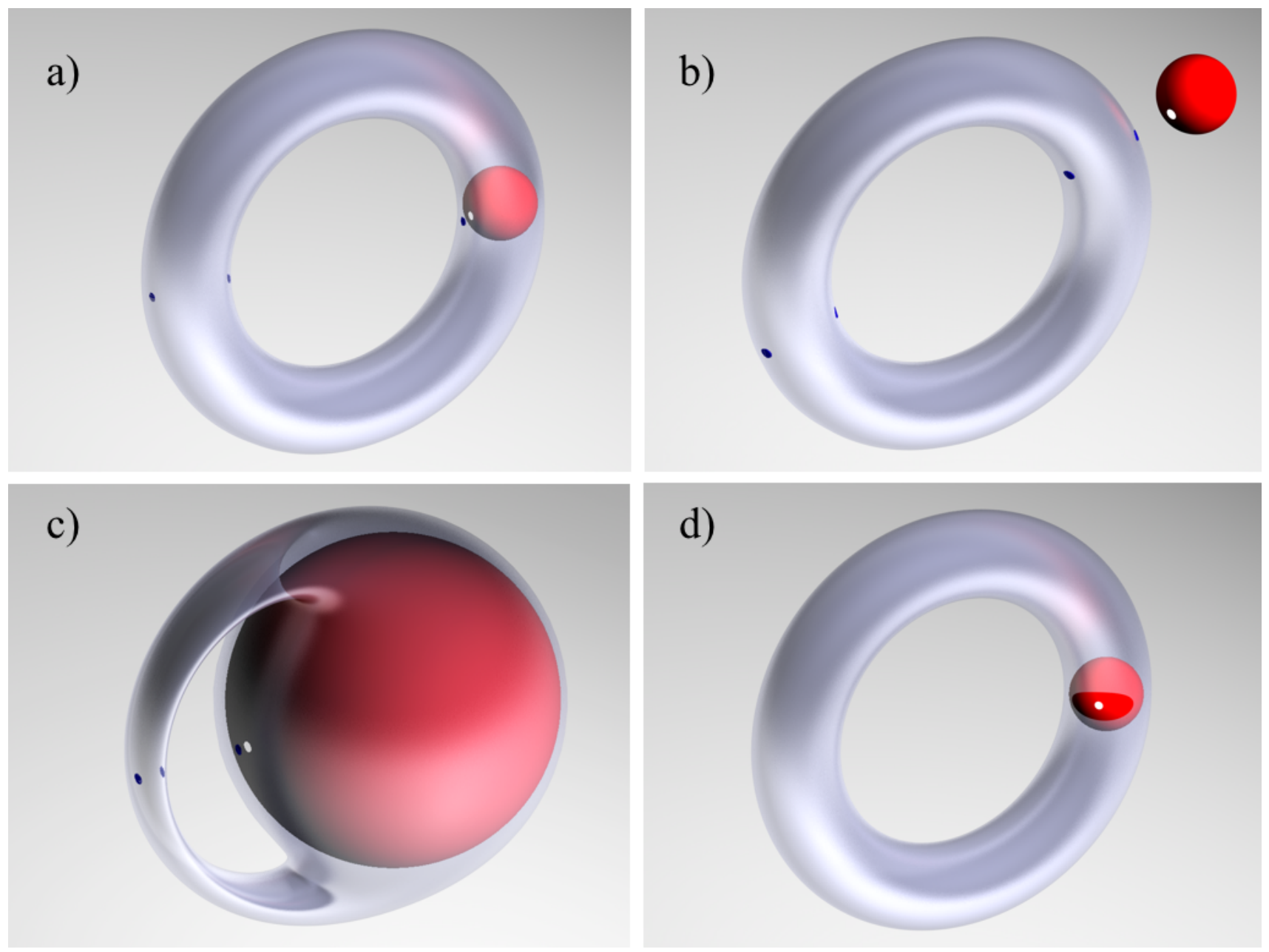

FIG. 6. (Color online) Visualization of the map $\mathcal{M}$ from the torus, i.e. the Brillouin zone, to the unit sphere of normalized spin expectation values. For the topologically non-trivial state (a), the sphere is placed inside the torus and the rays from the center of the sphere intersect the torus either once or three times. The south pole on the sphere is indicated by a white dot, and the ray through the south pole intersects the torus in the three blue dots. In the BZ these blue dots represent the $\boldsymbol{k}_{c}$, the centers of the three micro-vortices with vanishing $S_{z}$. For the topologically trivial state (b), the sphere is placed outside the torus and the rays from the center of the sphere intersect the torus either zero, two or four times. The topology of (c) is equivalent to that of (a); however the torus here is so much distorted by inflating the sphere that the multiple projection of the torus onto the sphere through $\mathcal{M}$, in the neighborhood of the south pole, is made particularly evident. In contrast, the characteristics of the map for finite temperature is sketchily represented by a sphere which pierces the torus in (d); here the intersecting torus and sphere are not related to a topological state; it is rather a candidate situation for finite temperature above a topological transition (see Sec. IV B).

four times (Fig. 6b) at the blue points. In Fig. 6c, the torus is so much distorted (by "inflating" the sphere) that the three sections of the torus, which are mapped onto the sphere in the neighborhood of the south pole, are made compellingly explicit: the rather flat areas of the "handle" produce the square-like grid in the vicinity of the south pole in Fig. 4d. These figures visualize that the map $\mathcal{M}$ is homotopically equivalent to $\hat{\boldsymbol{S}}_{\text {reg }}$. For $H_{z}>H_{\mathrm{t}}$ the entire sphere is located in the interior of the torus, while for $H_{z}<H_{\mathrm{t}}$ the sphere is outside the torus - see Figs. $6 \mathrm{a}$ and $6 \mathrm{~b}$, respectively.

It has to be emphasized that the meron number, Eq. (41), is a topological invariant only for the spe- cial class of singular maps presented here. Without the micro-vortices at the corners and face centers of the BZ, which are mapped to the equator, all spin textures with $S_{z} \geq 0$ everywhere are either topologically trivial $\left(N_{\mathrm{S}}=0\right)$, if the map $\hat{\boldsymbol{S}}$ is continuous, or $N_{\mathrm{S}}$ takes nonquantized real values, depending on the details of the texture, if $\hat{\boldsymbol{S}}$ is discontinuous but does not have the particular features described above. In the case that not the equator but some other curve in the upper hemisphere forms the limit set at the isolated singular points of the map $\hat{\boldsymbol{S}}$, the surface integral in Eq. (35) will not be a multiple of $2 \pi$. As will be discussed in the next section, this latter case indeed applies when the spin-expectation 


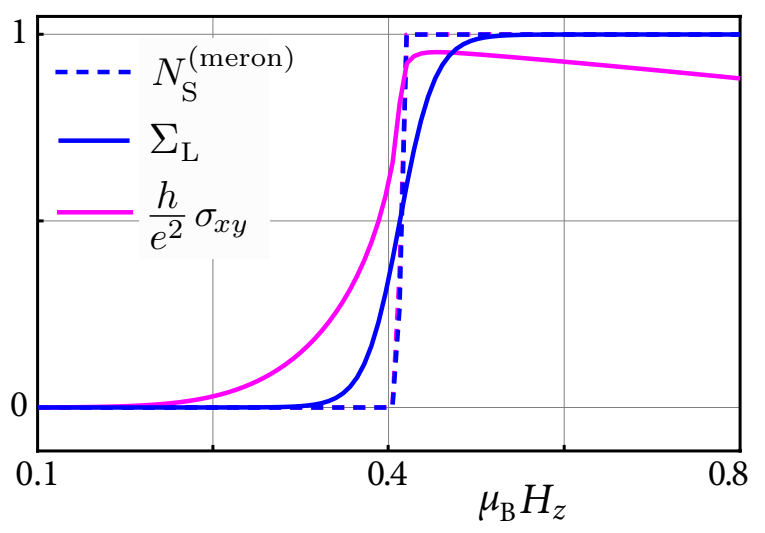

FIG. 7. (Color online) Comparison of Zeeman-field dependences for the meron number $N_{\mathrm{S}}^{(\text {meron })}=\frac{1}{2 \pi} \int_{B Z} \Omega_{\mathrm{S}}(\boldsymbol{k}) \mathrm{d}^{2} k$ (dashed line), the finite temperature equivalent $\Sigma_{\mathrm{L}}(T)$ (blue line), and the integral $C$ in the Kubo formula Eq. (20) (magenta line) for the Hall conductance. The two latter quantities are evaluated for $T=0.02 t$.

value is evaluated at finite temperatures.

\section{B. Out-of-plane magnetic field, $T>0$}

For finite temperatures, $T>0$, the evaluation of the spin-expectation values requires to take the trace over all states and thereby also the contributions from excited states are mixed in. We first assume that the temperature is smaller than the excitation gap to paired states on the upper, unoccupied $\xi_{k}^{+}$band with an order parameter of the opposite chirality; for the topological superconductor with $H_{z}>H_{\mathrm{t}}$ we therefore consider the temperature regime $k_{B} T \ll \mu_{B}\left(H_{z}-H_{\mathrm{t}}\right)$. In this case the contributions from excited states with a magnetization, which is lower than the ground-state magnetization in the external Zeeman field, are negligibly small. In essence, $S_{z}(T)$ is expected to increase with $T$ at these low temperatures. In particular, also the expectation values $S_{z}\left(\boldsymbol{k}_{c}\right)$ at the singular points on the BZ boundary turn finite and positive. The equator is therefore no longer reached by the map $\hat{\boldsymbol{S}}(\boldsymbol{k})$. For the map $\mathcal{M}$, this situation is sketchily represented in Fig. 6d by a sphere which pierces the torus with its south pole outside. This picture visualizes that an area around the south pole no longer has preimages on the torus for the map $\mathcal{M}$. In a strict sense, it is therefore only the superconducting state at zero temperature that is characterized by integer valued topological invariants which are based on its spin texture in momentum space.

We define a BZ-integral over the temperature dependent skyrmion-like density $\Omega_{\mathrm{L}(T>0)}(\boldsymbol{k})$, which is the finite temperature generalization of $\Omega_{\mathrm{L}}(\boldsymbol{k})$ from Eq. (37), through

$$
\Sigma_{\mathrm{L}}(T)=\frac{1}{2 \pi} \int_{B Z} \Omega_{\mathrm{L}(T>0)}(\boldsymbol{k}) \mathrm{d}^{2} k
$$

This quantity smoothly connects to the zero temperature limit with $\Sigma_{\mathrm{L}}(T=0)=N_{\mathrm{S}}^{(\text {meron })}=2 N_{\mathrm{S}}$ (cf. Eqs. (41) and (35)). Moreover, the Hall conductance $\sigma_{x y}(T)$ is well defined at finite temperature, and for $T \rightarrow 0$ the integrals $\frac{h}{e^{2}} \sigma_{x y}(T)$ and $\Sigma_{\mathrm{L}}(T)$ merge with the established invariants $C_{\mathrm{TKNN}}=N_{\mathrm{S}}^{(\text {meron })}$. The finite and zero temperature results are contrasted in Fig. 7.

At zero temperature the BZ-integral over the Berry curvature, the integral over the skyrmion density, and the Hall conductance, all yield a step-like behavior at $H_{z}=$ $H_{\mathrm{t}}$ (dashed step-function in Fig. 7). By contrast, the step is smoothed for finite temperature - however, qualitatively different for the skyrmion-like number $\Sigma_{\mathrm{L}}(T)$ and the Hall conductance. Even though $\Sigma_{\mathrm{L}}(T)$ well approaches the values 0 and 1 for $H_{z}$ away from $H_{z}=H_{\mathrm{t}}$, this quantity is not a topological invariant for finite temperature because such an invariant has to adopt these discrete values strictly. Yet, we emphasize that the physics at zero temperature is continuously approached for $T \rightarrow 0$, and the spin texture in momentum space maintains some of its qualitative features also at finite temperatures. This is suitably characterized by $\Sigma_{\mathrm{L}}(T)$.

Eventually, if the temperature is larger than the excitation gap to the states with opposite helicity $\left(k_{B} T \gtrsim\right.$ $\left.\mu_{B}\left(H_{z}-H_{\mathrm{t}}\right)\right)$, also states with spin $z$-component antiparallel to the applied field are thermally excited. However, for the relevant case of $H_{z}>H_{\mathrm{t}}$, these states are still separated by a small yet finite gap (except precisely at $H_{z}=H_{\mathrm{t}}$ ) from the states with opposite (positive) helicity and parallel alignment of their $z$-component. Therefore their thermal weight is smaller than that of states with negative helicity and their contribution is not sufficient to compensate the positive values of $S_{z}\left(\boldsymbol{k}_{c}\right)$. Correspondingly, the situation is similar to the case with $k_{B} T \ll \mu_{B}\left(H_{z}-H_{\mathrm{t}}\right)$.

At the topological transition $H_{z}=H_{\mathrm{t}}$ the energy gap closes and quantum critical behavior in the vicinity of this transition is expected $[13,55,56]$. In our work we focus exclusively on the mean-field solutions for the superconducting state; the intricate properties of the transition itself and the concomitant quantum critical behavior are not the scope of the present work and remain yet to be explored.

\section{In-plane magnetic field}

As indicated above, finite in-plane components of the magnetic field necessitate an explicit solution of the selfconsistency condition Eq. (27), including the search for the optimum COMM. A finite COMM is required, if the in-plane field exceeds a certain threshold value [37, 5153]. This threshold is reached when the indirect gap closes (see Ref. [37]). In fact, due to the slight difference in magnitude of the offset momenta $\boldsymbol{q}^{+}$and $\boldsymbol{q}^{-}$of the two Fermi surface sheets (see Fig. 2) at least two different COMMs are needed to optimize the intra-band 
(a) $H_{x}=0$

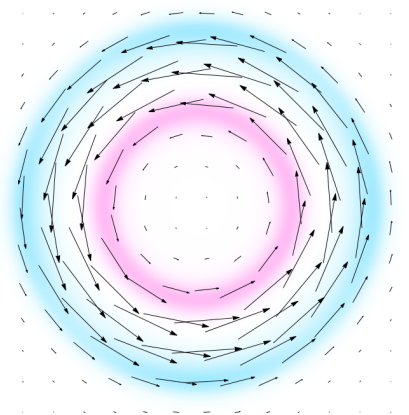

(b) $\mu_{B} H_{x}=0.15 t$

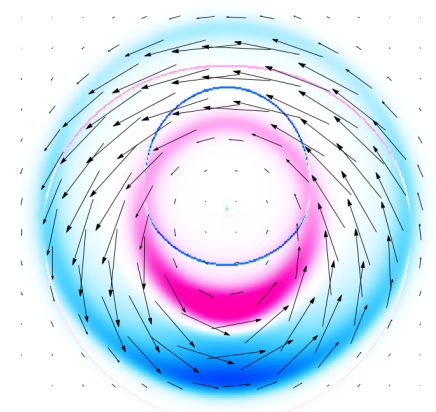

(c) $\mu_{B} H_{x}=0.5 t$

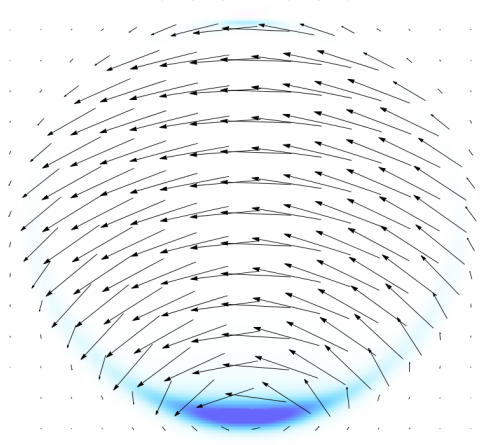

$-0.002$

0

$\Omega_{\mathrm{B}}(\boldsymbol{k})$

FIG. 8. (Color online) Vector-fields of the (unnormalized) in-plane spin-expectation values $\left(S_{x}(\boldsymbol{k}), S_{y}(\boldsymbol{k})\right)$ in the central part of the Brillouin zone for a constant out-of-plane field component $\left(\mu_{B} H_{z}=0.1 t\right)$ at zero temperature and increasing in-plane field strength: (a) $H_{x}=0$, (b) $\mu_{B} H_{x}=0.15 t$, and (c) $\mu_{B} H_{x}=0.5 t$. Here, the critical field strength for $\mu_{B} H_{x}$ to reach a topologically non-trivial superconducting state is approximately $0.42 t$. The Berry curvature is shown as a colored background. In (a) and (b) $N_{\mathrm{S}}^{(\text {meron })}=0$, while $N_{\mathrm{S}}^{(\text {meron })}=1$ in (c).

pairing in the $\xi_{\boldsymbol{k}}^{+}$and the $\xi_{\boldsymbol{k}}^{-}$band $[42,53]$.

For a finite in-plane field component we focus here on the case of zero temperature. A similar reasoning as in Sec. IV B also applies to tilted fields for finite temperatures. As shown in Ref. [37], the magnetic-field induced topological phase transition persists upon rotating the field orientation. Also for an almost in-plane field orientation, the momentum-space spin texture characteristically reflects the transition and the meron counting number $N_{\mathrm{S}}^{\text {(meron) }}$ discontinuously changes between 0 and \pm 1 upon increasing the field strength. The sign of the meron number is determined by the sign of $H_{z}$.

Beyond the critical field strength where only intraband pairing in the $\xi_{\boldsymbol{k}}^{-}$band with a single COMM $\boldsymbol{q}^{-}$ remains, the relation between $\Omega_{\mathrm{L}}(\boldsymbol{k})$ and the Berry curvature $\Omega_{\mathrm{B}}(\boldsymbol{k})$, Eq. (38), is modified to

$$
\Omega_{\mathrm{B}}(\boldsymbol{k})=\frac{1}{2}\left[\Omega_{\mathrm{L}}(\boldsymbol{k})+\Omega_{\mathrm{L}}\left(-\boldsymbol{k}+\boldsymbol{q}^{-}\right)\right] .
$$

This relation signifies that the Berry curvature $\Omega_{\mathrm{B}}(\boldsymbol{k})$ is a symmetrized version of $\Omega_{\mathrm{L}}(\boldsymbol{k})$ and both terms in the bracket of Eq. (44) integrate up to the same integer $N_{\mathrm{S}}^{\text {(meron) }}$. Equation (44) fails for a $\boldsymbol{k}$-dependent order parameter $\Delta_{\boldsymbol{q}}(\boldsymbol{k})$ because it will generally break the $\boldsymbol{k} \leftrightarrow$ $-\boldsymbol{k}+\boldsymbol{q}^{-}$symmetry.

Figure 8 shows the evolution of the in-plane spin textures with increasing in-plane field component. For $H_{x}=0$ the spin texture is qualitatively equivalent to the texture in Fig. 3b for the larger out-of-plane field component, but still $H_{z}<H_{\mathrm{t}}$. The colored shaded re- gions indicate the local Berry curvature as evaluated from $\Omega_{\mathrm{B}}(\boldsymbol{k})$. For $H_{x}=0$ the COMM of the electron pairs is zero and $\Omega_{\mathrm{B}}(\boldsymbol{k})=\Omega_{\mathrm{L}}(\boldsymbol{k})$.

For finite in-plane fields, but still below the critical field strength for the topological transition, the Fermi surface sheets of the $\xi_{\boldsymbol{k}}^{ \pm}$bands move off-center away from the $\Gamma$ point $\boldsymbol{k}=\mathbf{0}$ in opposite directions. Specifically for the example shown in Fig. 8b, i.e. $H_{x}>0, H_{y}=0$, the Fermi surface sheets move along the $k_{y}$-direction; the pairing on the $\xi_{\boldsymbol{k}}^{ \pm}$bands now requires finite COMMs $\boldsymbol{q}^{+}$and $\boldsymbol{q}^{-}$, respectively. The spin texture directly reflects this offcenter movement: the region in momentum space around which the in-plane spin components wind, shifts along the $k_{y}$-direction towards the BZ boundary. For $H_{x}=0$ and $\mu_{B} H_{x}=0.15 t$ in Figs. 8a and 8b, the compensating closed, fuzzy contours with Berry curvatures of opposite sign remain clearly visible; indeed for these two cases the BZ integral over the Berry curvature vanishes.

Instead, if $H_{x}$ exceeds a threshold field to enter the topologically non-trivial superconducting state, the sign of $\Omega_{\mathrm{B}}(\boldsymbol{k})$ is unique and its BZ integral leads to the first Chern number $C_{\mathrm{TKNN}}=\operatorname{sign}\left(H_{z}\right)$. This situation is realized for $\mu_{B} H_{x}=0.5 t$ with the spin texture in Fig. 8c. The unique sign of $\Omega_{\mathrm{B}}(\boldsymbol{k})$ is achieved, when the $\xi_{\boldsymbol{k}}^{+}$band energies are sufficiently raised by the tilted Zeeman field such that only the $\xi_{\boldsymbol{k}}^{-}$band states remain occupied. $\mathrm{Cu}-$ riously, the winding center for the in-plane spin components has shifted to the near vicinity of the normal-state Fermi surface of the $\xi_{k}^{-}$band. Of course, in the superconducting phase, a Fermi surface seizes to exist, but the 
(a) $H_{x}=0$

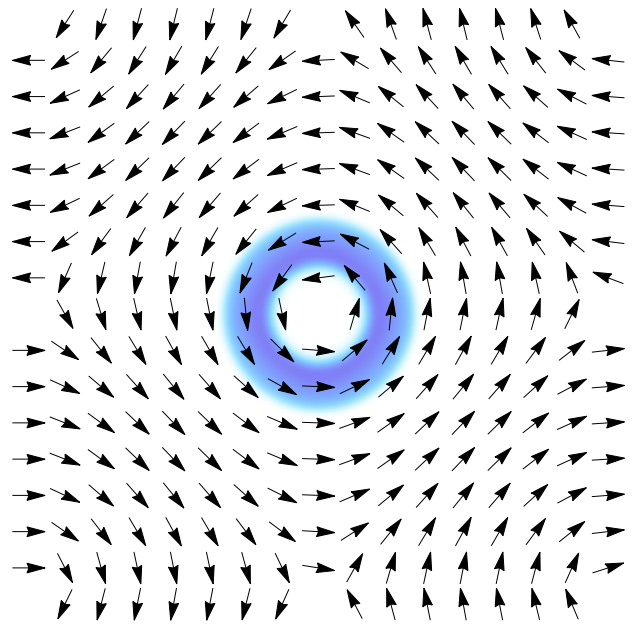

(b) $\mu_{B} H_{x}=0.5 t$
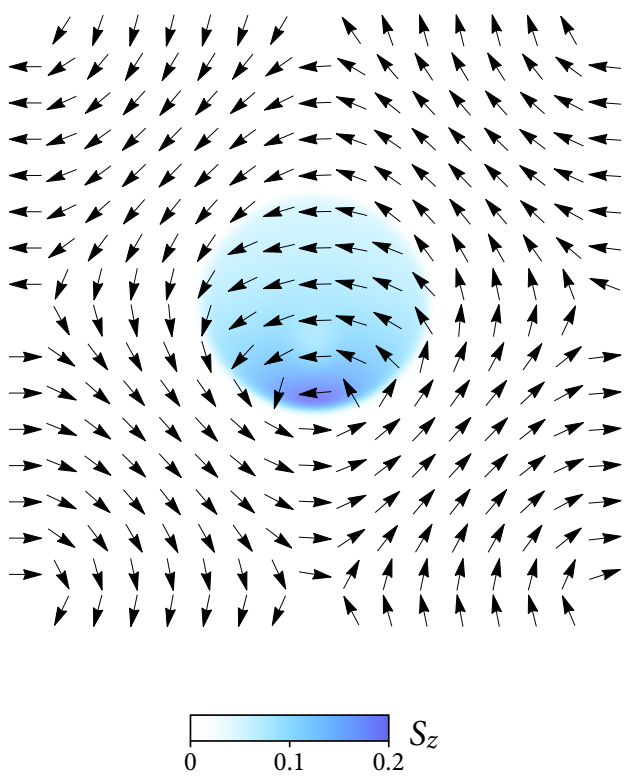

FIG. 9. (Color online) Vector fields of the normalized in-plane spin expectation-values, $\left(S_{x}(\boldsymbol{k}), S_{y}(\boldsymbol{k})\right) / \sqrt{S_{x}^{2}+S_{y}^{2}}$, in the BZ for (a) the topologically trivial state with zero magnetic field, and (b) the topologically non-trivial $s$-wave superconducting state with $\mu_{B} H_{x}=0.5 t$ and $\mu_{B} H_{z}=0.1 t$. The component $S_{z}(\boldsymbol{k})$ is shown as colored background density. The values of $k_{x}, k_{y}$ range from $-\pi$ to $\pi$. The critical value of $\mu_{B} H_{x}$ for the transition to the non-trivial topological state is approximately $\mu_{B} H_{x}=0.42 t$.

imprints of the normal state Fermi surfaces are clearly visible in the spin textures. In Figs. 8a and 8b, both the $\xi_{\boldsymbol{k}}^{+}$and the $\xi_{\boldsymbol{k}}^{-}$band are partially occupied, while in Fig. $8 \mathrm{c}$ the $\xi_{k}^{+}$band is empty.

In order to better trace the spin-vector field pattern in momentum space, the normalized in-plane spinexpectation values $\left(S_{x}(\boldsymbol{k}), S_{y}(\boldsymbol{k})\right) / \sqrt{S_{x}^{2}+S_{y}^{2}}$ are dis- played in Fig. 9a for the trivial topological phase with out-of-plane magnetic field $H_{z}<H_{\mathrm{t}}$, and in Fig. 9b for the topological phase induced by a sufficiently large tilted magnetic field $\left(\mu_{B} H_{x}=0.5 t\right)$. The transition to the topologically non-trivial $s$-wave superconducting state is found at approximately $\mu_{B} H_{x}=0.42 t$ for $\mu_{B} H_{z}=0.1 t$. The out-of-plane spin component $S_{z}(\boldsymbol{k})$ is represented by a colored background.

As already discussed in Sec. IV A we identify again four micro-vortices in momentum-space also for out-of-plane fields. It is instructive to count their vorticities $\mathcal{V}$ defined through the sign of an integral along a loop $\boldsymbol{k}(s)$ around the center of the vortex [57]:

$$
\mathcal{V}\left(\boldsymbol{k}_{c}\right)=\operatorname{sign}\left(\frac{1}{2 \pi} \int_{0}^{2 \pi} d s\left[\hat{\boldsymbol{S}}(\boldsymbol{k}(s)) \times \frac{d \hat{\boldsymbol{S}}(\boldsymbol{k}(s))}{d s}\right]_{z}\right) .
$$

The central vortex in Fig. 9a and the micro-vortex at the BZ corners $\boldsymbol{k}_{c}=( \pm \pi, \pm \pi)$ and $\boldsymbol{k}_{c}=(\mp \pi, \pm \pi)$ have vorticity +1 whereas the two micro-vortices at the BZ faces $\boldsymbol{k}_{c}=(0, \pm \pi)$ and $\boldsymbol{k}_{c}=( \pm \pi, 0)$ have each vorticity -1 . The total vorticity $\mathcal{V}_{\mathrm{t}}$ is zero. In fact, for periodic boundary conditions, it is a necessary requirement to have $\mathcal{V}_{\mathrm{t}}=0$, i.e., for a $\mathrm{BZ}$ represented by a torus. This implies, for example, that the central vortex cannot appear alone. Indeed, from the fact that the torus is mapped by $\mathcal{M}$ to a unit sphere it is evident that four micro-vortices must exist which are mapped to the south pole of the sphere (see Fig. 6b).

For the topologically non-trivial $s$-wave superconducting state in a tilted magnetic field (see Fig. 9b), the micro-vortices at the BZ boundary are preserved. The central vortex, however, is moved towards the normal state Fermi surface in the direction perpendicular to the in-plane magnetic field (as discussed in connection with Fig. 8). But the center of the central vortex never traverses the normal-state Fermi surface. The vortex center has the maximum $S_{z}$ and is therefore mapped by $\mathcal{M}$ to the north pole of the sphere for magnetic fields larger than the critical field, but the vorticity of this spin pattern is preserved and the total vorticity remains zero. The important observation here is that the central vortex is trapped inside the Fermi surface when the superconducting gap is finite. In the normal state at small temperature the central vortex glides outside the Fermi surface and moves to the BZ boundary for $H_{x}>\alpha\left|\sin k_{y, \mathrm{~F}}\right|$, where $k_{y, \mathrm{~F}}$ is the Fermi momentum in the direction of $(0,-\pi)$. The spin field vortex pattern is thereby annihilated. Whether a similar trapping of the central vortex is possible also in a nodal superconductor may be yet another issue for future work on momentum-space spin textures.

\section{CONCLUSION}

As we have demonstrated for the specific example of a topological $s$-wave superconductor, the spin texture in 
momentum space provides an alternative tool to identify a system's topological character. The meron counting number is a well-defined topological invariant; its discontinuous change signifies a transition between topologically distinct ground states. For the meron number to be a topological invariant, it proves crucial that the spin texture has isolated singular points at the BZ boundary. These singularities control the essential maps from the compact manifold of the BZ to the unit sphere of normalized spin-expectation values and ultimately allow for a finite, integer valued meron number.

For time-reversal invariant topological insulators it was shown in Sec. II that the skyrmion counting number of the spin texture is an integer valued topological invariant, equivalent to the Brouwer degree of mapping or the first Chern number, which is the BZ integral over the Berry curvature. The topological $s$-wave superconductor relies on the Zeeman coupling to an external magnetic field. This time-reversal symmetry breaking magnetic field prevents the spin map to cover the full spin unit sphere and therefore necessitates to turn to integer valued meron or half-integer valued skyrmion numbers. The natural question still has to be answered, whether this paradigm applies to topological insulators with broken time-reversal symmetry, too.

An example, in this context, is the emergence of a hedgehog spin-texture in momentum space in magnetically doped 3D topological insulators [58]. Also on the surface of a 3D topological insulator, if an exchange field is applied, momentum-space hedgehog or skyrmion textures emerge [59], where the former has skyrmion number $1 / 2$.

In contrast to the ground state concepts of Berry curvature or Chern number, the topological fingerprint in a system's spin texture may allow a natural extension to finite temperatures. As we have shown for the topological $s$-wave superconductor, the spin expectation values at finite temperatures already reveal signatures of the groundstate with finite integer valued topological invariants. This raises the general question, to what extent topological phase transitions influence the electronic properties at finite temperatures near the critical control parameters for the topological transition. The spin textures, which we have encountered and analyzed in this work, appear to offer an example for such a phenomenon, and remains yet to be translated into a broader context.

\section{ACKNOWLEDGEMENTS}

We gratefully acknowledge Alexander Herrnberger's support in the design of figure 6 . This work was supported by the DFG through TRR 80 .

\section{Appendix A: Brouwer degree and skyrmion number}

In this section we derive Eqs. (9) and (12) of the main text. The domain of the map $\boldsymbol{h}(\boldsymbol{k})$ is $T^{2}$ and a subset of $\mathbb{R}^{3}$ is its range. This subset is homeomorphic to a subset of $S^{2}$, the range of the normalized map $\boldsymbol{h} / h$. Both $\boldsymbol{h}$ and $\boldsymbol{h} / h$ have the same Brouwer degree.

We select one of the points $\boldsymbol{k}_{l}$, say $\boldsymbol{k}_{0}$, and-for convenience shift coordinates such that $\boldsymbol{k}_{0}=\mathbf{0}$. Then $h(\mathbf{0})=h_{z}(\mathbf{0})$, and $h_{x}(\mathbf{0})=h_{y}(\mathbf{0})=0$. The two components of the map, $h_{x}\left(k_{1}, k_{2}\right)$ and $h_{y}\left(k_{1}, k_{2}\right)$, are linearized around $\boldsymbol{k}=\mathbf{0}$ as

$$
h_{x}=J_{11} k_{1}+J_{12} k_{2}, \quad h_{y}=J_{21} k_{1}+J_{22} k_{2},
$$

where $J_{i k}$ denotes the elements of the Jacobi matrix

$$
\hat{J}\left(\boldsymbol{k}_{0}\right)=\left.\frac{\partial\left(h_{x}, h_{y}\right)}{\partial\left(k_{1}, k_{2}\right)}\right|_{\boldsymbol{k}_{0}}
$$

at the point $\boldsymbol{k}_{0}$, i.e. the differential of the map from $T^{2}$ into $\mathbb{R}^{3}$ given by $\boldsymbol{h}(\boldsymbol{k})$, where we have chosen $h_{x}(\boldsymbol{k}), h_{y}(\boldsymbol{k})$ as local coordinates of the image manifold. We thereby find

$$
\begin{gathered}
R^{2}\left(k_{1}, k_{2}\right)=h_{x}^{2}+h_{y}^{2}+\mathcal{O}\left(k_{j}^{4}\right), \\
v_{1}=\frac{J\left(\boldsymbol{k}_{0}\right)}{h_{x}^{2}+h_{y}^{2}} k_{2}, \quad v_{2}=-\frac{J\left(\boldsymbol{k}_{0}\right)}{h_{x}^{2}+h_{y}^{2}} k_{1},
\end{gathered}
$$

with the Jacobian $J\left(\boldsymbol{k}_{0}\right)=\operatorname{det} \hat{J}\left(\boldsymbol{k}_{0}\right)$. To evaluate $\oint_{c_{l}} \boldsymbol{v}$ for a small circle $c_{0}$ with radius $r$ around $\boldsymbol{k}_{0}=\mathbf{0}$, we set $k_{1}=r \cos \psi, k_{2}=r \sin \psi$. With $\mathrm{d} s=\left(-k_{2} \mathrm{~d} \psi, k_{1} \mathrm{~d} \psi\right)^{\top}$, we obtain

$$
\oint_{c_{0}} \boldsymbol{v}=-J\left(\boldsymbol{k}_{0}\right) r^{2} \int_{0}^{2 \pi} \frac{\mathrm{d} \psi}{h_{x}^{2}(r, \psi)+h_{y}^{2}(r, \psi)} .
$$

We write the denominator of Eq. (A5) as

$$
h_{x}^{2}+h_{y}^{2}=\boldsymbol{k}^{\top} \hat{J}^{\top}\left(\boldsymbol{k}_{0}\right) \hat{J}\left(\boldsymbol{k}_{0}\right) \boldsymbol{k}
$$

where the quadratic form $\hat{J}^{\top} \hat{J}$ can be diagonalized by an orthogonal transformation. With the eigenvalues $\lambda_{1,2}$ of $\hat{J}\left(\boldsymbol{k}_{0}\right)$ and upon rotating $\psi$ accordingly, we are lead to

$$
h_{x}^{2}+h_{y}^{2}=r^{2}\left(\lambda_{1}^{2} \cos ^{2} \psi+\lambda_{2}^{2} \sin ^{2} \psi\right) .
$$

Performing the elementary integral over the angle $\psi$ we find

$$
\oint_{c_{0}} \boldsymbol{v}=-2 \pi \frac{J\left(\boldsymbol{k}_{0}\right)}{\left|\lambda_{1} \lambda_{2}\right|}=-2 \pi \operatorname{sign}\left(J\left(\boldsymbol{k}_{0}\right)\right) .
$$

Upon collecting all preimages $\boldsymbol{k}_{l}$ of $\left(0,0, h_{z}\right)$ for arbitrary $h_{z}$, i.e. all singular points of $\boldsymbol{v}$, the final result reads

$$
\int_{B Z} \boldsymbol{\Omega}=2 \pi \sum_{l} \operatorname{sign}\left(J\left(\boldsymbol{k}_{l}\right)\right)=2 \pi C_{\text {Brouwer }} .
$$

To derive Eq. (12), we evaluate (10) using (5) and obtain 


$$
\Omega=2 R^{-2}\left[\left(\partial_{1} h_{y}\right)\left(\partial_{2} h_{x}\right)-\left(\partial_{1} h_{x}\right)\left(\partial_{2} h_{y}\right)\right]+\left(h_{y} \partial_{2} h_{x}-h_{x} \partial_{2} h_{y}\right) \partial_{1}\left(R^{-2}\right)-\left(h_{y} \partial_{1} h_{x}-h_{x} \partial_{1} h_{y}\right) \partial_{2}\left(R^{-2}\right)
$$

The terms in Eq. (A10), which contain derivatives of $R^{-2}$, can be combined into

$$
\frac{2}{h R^{4}}\left\{\left(h_{x}^{2}+h_{y}^{2}\right)\left(2 h-h_{z}\right) J_{x y}+\left(h-h_{z}\right)^{2}\left[h_{x} J_{y z}+h_{y} J_{z x}\right]\right\}
$$

where we have used the notation Eq. (11) in the main text. The expression in (A11) is still divergent at the points $\boldsymbol{k}_{l}$ where $R$ vanishes. However, together with the first term in Eq. (A10) we find

$$
\begin{aligned}
\Omega= & J_{x y}\left[\frac{2}{h R^{4}}\left(2 h-h_{z}\right)\left(h_{x}^{2}+h_{y}^{2}\right)-\frac{2}{R^{2}}\right] \\
& +\frac{2}{h R^{4}}\left(h-h_{z}\right)^{2}\left[h_{x} J_{y z}+h_{y} J_{z x}\right],
\end{aligned}
$$

which yields after some simplifications Eq. (12).

To show that $N_{S}=C_{\text {Brouwer }}$, we first write $\boldsymbol{h}(\boldsymbol{k})$ in spherical coordinates: $\boldsymbol{h}(\boldsymbol{k})=h(\boldsymbol{k}) \boldsymbol{e}_{r}(\vartheta(\boldsymbol{k}), \varphi(\boldsymbol{k}))$, and

$$
\partial_{j} \boldsymbol{h}=\left(\partial_{j} h\right) \boldsymbol{e}_{r}+h\left(\partial_{j} \vartheta\right) \boldsymbol{e}_{\vartheta}+h \sin \vartheta\left(\partial_{j} \varphi\right) \boldsymbol{e}_{\varphi}
$$

The cross product in the skyrmion density Eq. (14) then takes the form

$$
\begin{aligned}
\partial_{1} \boldsymbol{h} & \times \partial_{2} \boldsymbol{h}=h^{2} \sin \vartheta\left(\partial_{1} \vartheta \partial_{2} \varphi-\partial_{1} \varphi \partial_{2} \vartheta\right) \boldsymbol{e}_{r} \\
& +h \sin \vartheta\left(\partial_{1} \varphi \partial_{2} h-\partial_{1} h \partial_{2} \varphi\right) \boldsymbol{e}_{\vartheta} \\
& +h\left(\partial_{1} h \partial_{2} \vartheta-\partial_{1} \vartheta \partial_{2} h\right) \boldsymbol{e}_{\varphi}
\end{aligned}
$$

and therefore

$$
\Omega_{\mathrm{h}}(\boldsymbol{k})=\sin \vartheta\left[\left(\partial_{1} \vartheta\right)\left(\partial_{2} \varphi\right)-\left(\partial_{1} \varphi\right)\left(\partial_{2} \vartheta\right)\right] .
$$

Note that the derivatives of $h(\boldsymbol{k})$ have dropped out in Eq. (A15). This implies

$$
\Omega_{\mathrm{h}}(\boldsymbol{k})=\frac{\boldsymbol{h}}{h^{3}} \cdot\left[\partial_{1} \boldsymbol{h} \times \partial_{2} \boldsymbol{h}\right]=\hat{\boldsymbol{h}} \cdot\left[\partial_{1} \hat{\boldsymbol{h}} \times \partial_{2} \hat{\boldsymbol{h}}\right],
$$

with the normalized $\hat{\boldsymbol{h}}=\boldsymbol{h} / \boldsymbol{h}$. The right hand side of Eq. (A15) is $\sin \vartheta(\boldsymbol{k})$ times the Jacobian

$$
J(\boldsymbol{k})=\frac{\partial(\vartheta, \varphi)}{\partial\left(k_{1}, k_{2}\right)}
$$

of the map $\hat{\boldsymbol{h}}(\boldsymbol{k})$ from the torus to the unit sphere. For $N_{\mathrm{S}}$ of Eq. (15) we eventually find

$4 \pi N_{\mathrm{S}}=\int_{B Z} \sin \vartheta(\boldsymbol{k}) J(\boldsymbol{k}) \mathrm{d}^{2} k=N_{\mathrm{B}} \int_{S^{2}} \sin \vartheta \mathrm{d} \vartheta \mathrm{d} \varphi=4 \pi N_{\mathrm{B}}$

where $N_{\mathrm{B}} \in \mathbb{Z}$ counts the number of times each (regular) point of $S^{2}$ is attained by the map $\hat{\boldsymbol{h}}(\boldsymbol{k})$; the sign of $N_{\mathrm{B}}$ reflects the orientation of the map. $N_{\mathrm{S}}$ is therefore again the Brouwer degree of $\hat{\boldsymbol{h}}(\boldsymbol{k})$ and Eq. (A18) is equivalent to Eq. (9) because the surface form $\sin \vartheta \mathrm{d} \vartheta \wedge \mathrm{d} \varphi$ has the same orientation as $\mathrm{d} h_{x} \wedge \mathrm{d} h_{y}$ in the vicinity of the singular points with $\hat{\boldsymbol{h}}=\boldsymbol{e}_{z}$.

Yet another possibility to compute the skyrmion number $N_{\mathrm{S}}$ is furnished by the quantity

$$
\overline{\boldsymbol{S}_{\lambda}}=\left(\frac{h_{x}}{\bar{h}}, \frac{h_{y}}{\bar{h}}, \lambda \frac{h_{z}}{h}\right)
$$

for arbitrary real $\lambda$ with $\bar{h}=\sqrt{h_{x}^{2}+h_{y}^{2}}$. To verify this option, we begin with $\lambda=1$ and note that $\overline{\boldsymbol{S}}_{1}$ is the horizontal projection of the point $\hat{\boldsymbol{h}}=$ $(\cos \varphi \sin \vartheta, \sin \varphi \sin \vartheta, \cos \vartheta)$ on the unit sphere, onto the point $(\cos \varphi, \sin \varphi, \cos \vartheta)$ on the cylinder with unit barrel radius. Since the surface elements $\sin \vartheta \mathrm{d} \vartheta \wedge \mathrm{d} \varphi$ of the sphere and $\mathrm{d} \varphi \wedge \mathrm{d} z=\mathrm{d} \varphi \wedge \mathrm{d}(\cos \vartheta)$ of the cylinder are equivalent, we have

$$
\overline{\boldsymbol{S}}_{1} \cdot\left[\partial_{1} \overline{\boldsymbol{S}}_{1} \times \partial_{2} \overline{\boldsymbol{S}}_{1}\right]=\frac{\partial(\varphi, z)}{\partial\left(k_{1}, k_{2}\right)}=\sin \vartheta J(\boldsymbol{k}) .
$$

where

$$
\overline{\boldsymbol{S}}_{1}=\left(\frac{h_{x}}{\bar{h}}, \frac{h_{y}}{\bar{h}}, \frac{h_{z}}{h}\right)=\left(\frac{S_{x}}{\bar{S}}, \frac{S_{y}}{\bar{S}}, \frac{S_{z}}{S}\right)
$$

with $\bar{S}=\sqrt{S_{x}^{2}+S_{y}^{2}}$ and $S=\sqrt{S_{x}^{2}+S_{y}^{2}+S_{z}^{2}}$. Here the spin expectation values have been introduced as in Eq. (17).

The equality (A20) implies

$$
N_{\mathrm{S}}=\frac{1}{4 \pi} \int_{B Z} \overline{\boldsymbol{S}}_{1} \cdot\left[\partial_{1} \overline{\boldsymbol{S}}_{1} \times \partial_{2} \overline{\boldsymbol{S}}_{1}\right] \mathrm{d}^{2} k .
$$

As the surface element of the cylinder scales with $\lambda$, we immediately generalize to arbitrary $\lambda$

$$
\overline{\boldsymbol{S}}_{\lambda} \cdot\left[\partial_{1} \overline{\boldsymbol{S}}_{\lambda} \times \partial_{2} \overline{\boldsymbol{S}}_{\lambda}\right]=\lambda \sin \vartheta J(\boldsymbol{k}) .
$$

With the Berry curvature, written in this geometry,

$$
\Omega_{\lambda}(\boldsymbol{k})=\overline{\boldsymbol{S}}_{\lambda} \cdot\left[\partial_{1} \overline{\boldsymbol{S}}_{\lambda} \times \partial_{2} \overline{\boldsymbol{S}}_{\lambda}\right],
$$

the skyrmion number is reexpressed as

$$
N_{\mathrm{S}}=\frac{1}{4 \pi \lambda} \int_{B Z} \overline{\boldsymbol{S}}_{\lambda} \cdot\left[\partial_{1} \overline{\boldsymbol{S}}_{\lambda} \times \partial_{2} \overline{\boldsymbol{S}}_{\lambda}\right] \mathrm{d} k_{1} \mathrm{~d} k_{2} .
$$

\section{Appendix B: Pairing amplitudes in the helicity basis}

With the shorthand notation $h(\boldsymbol{k})^{-}=h_{x}(\boldsymbol{k})-i h_{y}(\boldsymbol{k})$ and $h_{z}=h_{z}(\boldsymbol{k})$ the intra- and inter-band pairing amplitudes in the helicity basis are explicitly given by 


$$
\begin{aligned}
& \Delta^{++}(\boldsymbol{k}, \boldsymbol{q})=\frac{\Delta_{\boldsymbol{q}}}{2 \sqrt{h(\boldsymbol{k}) h(-\boldsymbol{k}+\boldsymbol{q})}}\left\{\sqrt{\frac{h(\boldsymbol{k})+h_{z}}{h(-\boldsymbol{k}+\boldsymbol{q})+h_{z}}} h^{-}(-\boldsymbol{k}+\boldsymbol{q})-\sqrt{\frac{h(-\boldsymbol{k}+\boldsymbol{q})+h_{z}}{h(\boldsymbol{k})+h_{z}}} h^{-}(\boldsymbol{k})\right\} \\
& \Delta^{--}(\boldsymbol{k}, \boldsymbol{q})=\frac{\Delta_{\boldsymbol{q}}}{2 \sqrt{h(\boldsymbol{k}) h(-\boldsymbol{k}+\boldsymbol{q})}}\left\{\sqrt{\frac{h(-\boldsymbol{k}+\boldsymbol{q})-h_{z}}{h(\boldsymbol{k})-h_{z}}} h^{-}(\boldsymbol{k})-\sqrt{\frac{h(\boldsymbol{k})-h_{z}}{h(-\boldsymbol{k}+\boldsymbol{q})-h_{z}}} h^{-}(-\boldsymbol{k}+\boldsymbol{q})\right\}, \\
& \Delta^{+-}(\boldsymbol{k}, \boldsymbol{q})=\frac{\Delta_{\boldsymbol{q}}}{2 \sqrt{h(\boldsymbol{k}) h(-\boldsymbol{k}+\boldsymbol{q})}}\left\{\sqrt{\frac{h(\boldsymbol{k})+h_{z}}{h(-\boldsymbol{k}+\boldsymbol{q})-h_{z}}} h^{-}(-\boldsymbol{k}+\boldsymbol{q})+\sqrt{\frac{h(-\boldsymbol{k}+\boldsymbol{q})-h_{z}}{h(\boldsymbol{k})+h_{z}} h^{-}(\boldsymbol{k})}\right\}, \\
& \Delta^{-+}(\boldsymbol{k}, \boldsymbol{q})=\frac{-\Delta_{\boldsymbol{q}}}{2 \sqrt{h(\boldsymbol{k}) h(-\boldsymbol{k}+\boldsymbol{q})}}\left\{\sqrt{\frac{h(-\boldsymbol{k}+\boldsymbol{q})+h_{z}}{h(\boldsymbol{k})-h_{z}}} h^{-}(\boldsymbol{k})+\sqrt{\frac{h(\boldsymbol{k})-h_{z}}{h(-\boldsymbol{k}+\boldsymbol{q})+h_{z}}} h^{-}(-\boldsymbol{k}+\boldsymbol{q})\right\} .
\end{aligned}
$$

Note that in general $\Delta^{+-}(\boldsymbol{k}, \boldsymbol{q}) \neq \Delta^{-+}(\boldsymbol{k}, \boldsymbol{q})$. Only for the special $H_{z}=h_{z}=0$, i.e. for an in-plane magnetic field, $\Delta^{+-}(\boldsymbol{k}, \boldsymbol{q})=\Delta^{-+}(\boldsymbol{k}, \boldsymbol{q})$ and $\Delta^{+-}(\boldsymbol{k}, \boldsymbol{q})$ is even with respect to interchanging $\boldsymbol{k}$ and $-\boldsymbol{k}+\boldsymbol{q}$.
[1] D. J. Thouless, Topological Quantum Numbers in Nonrelativistic Physics, World Scientific, Singapore (1998).

[2] B. A. Bernevig, Topological Insulators and Topological Superconductors, Princeton Univ Press, Princeton (2013).

[3] M. Z. Hasan and C. L. Kane, Rev. Mod. Phys. 82, 3045 (2010).

[4] X.-L. Qi and S.-C. Zhang, Rev. Mod. Phys. 83, 1057 (2011).

[5] Z. F. Ezawa, Quantum Hall Effects, $3^{\text {rd }}$ Edition, World Scientific, Singapore (2013).

[6] D. J. Thouless, M. Kohmoto, M. P. Nightingale, and M. den Nijs, Phys. Rev. Lett. 49, 405 (1982).

[7] M. Kohmoto, Ann. Phys. (NY) 60, 343 (1985).

[8] M. Sato, Y. Takahashi, and S. Fujimoto, Phys. Rev. Lett. 103, 020401 (2009).

[9] J. Alicea, Phys. Rev. B 81, 125318 (2010).

[10] M. Sato, Y Takahashi, and S. Fujimoto, Phys. Rev. B 82, 134521 (2010).

[11] J. D. Sau, R. M. Lutchyn, S. Tewari, and S. Das Sarma, Phys. Rev. Lett. 104, 040502 (2010).

[12] S. Tewari, T. D. Stanescu, J. D. Sau, and S. Das Sarma, New J. Phys. 13, 065004 (2011).

[13] M. Gong, G. Chen, S. Jia, and C. Zhang, Phys. Rev. Lett. 109, 105302 (2012).

[14] J. Zhou, W. Zhang, and W. Yi, Phys. Rev. A 84, 063603 (2011).

[15] K. Seo, L. Han, and C. Sa de Melo, Phys. Rev. A 85 , 033601 (2012).

[16] H. Hu, L. Jiang, X. Liu, and H. Pu, Phys. Rev. Lett. 107, 195304 (2011).

[17] C. Qu, Z. Zheng, M. Gong, Y. Xu, L. Mao, X. Zou, G. Guo, and C. Zhang Nat. Commun. 4, 2710 (2013).

[18] W. Zhang and W. Yi, Nat. Commun. 4, 2711 (2013).

[19] C. Chen, Phys. Rev. Lett. 111, 235302 (2013).

[20] C. L. Kane and E. J. Mele, Phys. Rev. Lett. 95, 146802 (2005).

[21] C. L. Kane and E. J. Mele, Phys. Rev. Lett. 95, 226801 (2005).

[22] J. E. Moore and L. Balents, Phys. Rev. B 75, 121306(R) (2017).
[23] L. Fu, C. L. Kane, and E. J. Mele, Phys. Rev. Lett. 98, 106803 (2007).

[24] R. Roy, arXiv:0803.2868 (unpublished.

[25] A. Kitaev, AIP Conf. Proc. 1134, 22 (2009).

[26] X.-L. Qi, T. L. Huges, S. Raghu, and S.-C. Zhang, Phys. Rev. Lett. 102, 187001 (2009).

[27] M. Sato and S. Fujimoto, Phys. Rev. B 79, 094504 (2009).

[28] K. Björnson and A. M. Black-Schaffer, Phys. Rev. B 89, 134518 (2014).

[29] G. E. Volovik, The Universe in a Helium Droplet, Clarendon Press, Oxford (2003).

[30] J. Jost, Riemannian Geometry and Geometric Analysis (Springer, New York, 2005), p. 111.

[31] R. Bott and L. W. Tu, Differential Forms in Algebraic Topology (Springer, New York, 1982), p. 31.

[32] Note the missing factor $\sqrt{-1}$ in the definition of the Chern class as compared to [30], p.127. This is due to our definition of the connection $\boldsymbol{v}$ as real-valued in Eq.(3).

[33] D. Xiao, M.-C. Chang, and Q. Niu, Rev. Mod. Phys. 82, 1959 (2010).

[34] J. W. Milnor, Topology from the Differentiable Viewpoint (Princeton University Press, Princeton, 1965), p. 27.

[35] X. L. Qi, Y. S. Wu, and S. C. Zhang, Phys. Rev. B 74, 085308 (2006).

[36] B. A. Bernevig, T. L. Hughes, and S. C. Zhang, Science 314, 1757 (2006).

[37] F. Loder, A. P. Kampf, and T. Kopp, Sci. Rep. 5, 15302 (2015).

[38] M. Sigrist, AIP Conf. Proc. 1162, 55 (2009).

[39] L. Fu and C. L. Kane, Phys. Rev. Lett. 100, 096407 (2016).

[40] D. Vollhardt and P. Wölfle, The Superfluid Phases of Helium 3, Dover Publications Inc., Dover, 2013.

[41] M. Sigrist, A. Furusaki, C. Honerkamp, M. Matsumoto, K.K. Ng, and Y. Okuno, J. Phys. Soc. Jpn. 69, 127 (1999).

[42] F. Loder, A. P. Kampf, and T. Kopp, J. Phys.: Condens. Matter 25, 362201 (2013).

[43] A. A. Zyuzin, D. Rainis, J. Klinovaja, and D. Loss, Phys. Rev. Lett. 111, 056802 (2013). 
[44] C. L. M. Wong, J. L. Liu, K. T. Law, and P. A. Lee, Phys. Rev. B 88, 060504(R) (2013).

[45] F. Loder, A. P. Kampf, and T. Kopp, Phys. Rev. B 81, 020511(R) (2010).

[46] M. König, H. Buhmann, L. W. Molenkamp, T. Hughes, C.-X. Liu, X.-L. Qi, and S.-C. Zhang, J. Phys. Soc. Jpn. 77, 031007 (2008).

[47] Y. Dong, L. Dong, M. Gong, and H. Pu, Nat. Comm. 6, 6103 (2015).

[48] N. Nagaosa and Y. Tokura, Nat. Nanotech. 8899 (2013)

[49] A. I. Larkin and Yu. N. Ovchinnikov, Zh. Eskp. Teor. Fiz. 47, 1136 (1964) [Sov. Phys. JETP 20, 762 (1965)].

[50] P. Fulde and R. A. Ferrell, Phys. Rev. 135, A550 (1964).

[51] V. Barzykin and L. P. Gor'kov, Phys. Rev. Lett. 89, 227002 (2002).

[52] R. P. Kaur, D. F. Agterberg, and M. Sigrist, Phys. Rev. Lett. 94, 137002 (2005).

[53] K. Michaeli, A. C. Potter, and P. A. Lee, Phys. Rev. Lett. 108, 117003 (2012).

[54] Y. Xu and C. Zhang, Phys. Rev. Lett. 114, 110401 (2015).

[55] For a discussion of quantum critical behavior at the topological phase transition between an insulator and a Weyl semimetal see: B. J. Yang, E. G. Moon, H. Isobe, and N. Nagaosa, Nat. Phys. 10, 774 (2014).

[56] C. Castelnovo, S. Trebst, and M. Troyer, in Understanding Quantum Phase Transitions, edited by L. D. Carr, CRC Press, Tayler and Francis, 2010, p. 169; arXiv:0912.3272.

[57] We emphasize that $\mathcal{V}$ is not the winding number but rather distinguishes vortex and anti-vortex. The definition for the vorticity is valid for an $x y$-model even without the enclosing sign-function. Here we need the signfunction for a loop in the vicinity of the central vortex in the topologically non-trivial state where the spins have a sizable $S_{z}$ expectation value.

[58] S.-Y. Xu, M. Neupane, C. Liu, D. Zhang, A. Richardella, L. Andrew Wray, N. Alidoust, M. Leandersson, T. Balasubramanian, J. Sanchez-Barriga, O. Rader, G. Landolt, B. Slomski, J. Hugo Dil, J. Osterwalder, T.-R. Chang, H.T. Jeng, H. Lin, A. Bansil, N. Samarth, and M.-Z. Hasan, Nat. Phys. 8, 616 (2012).

[59] N. Mohanta, A. P. Kampf, and T. Kopp, arXiv:1607.04493v2 (2016). 